\title{
Physiological and Biochemical Mechanisms of Exogenously Applied Selenium for Alleviating Destructive Impacts Induced by Salinity Stress in Bread Wheat
}

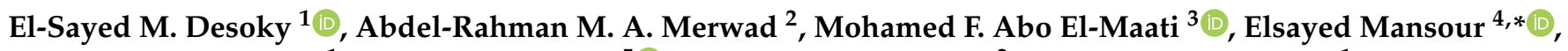 \\ Safaa M. A. I. Arnaout ${ }^{1}$, Mohamed F. Awad ${ }^{5}{ }^{-}$, Mohamed F. Ramadan ${ }^{3}$ and Seham A. Ibrahim ${ }^{1}$ \\ 1 Botany Department, Faculty of Agriculture, Zagazig University, Zagazig 44519, Egypt; \\ desoky_s@yahoo.com (E.-S.M.D.); safaamourad22@gmail.com (S.M.A.I.A.); \\ mohamedelbkh@yahoo.com (S.A.I.) \\ 2 Soil Science Department, Faculty of Agriculture, Zagazig University, Zagazig 44519, Egypt; \\ amerwad@yahoo.com \\ 3 Agricultural Biochemistry Department, Faculty of Agriculture, Zagazig University, Zagazig 44519, Egypt; \\ aboelmaati@yahoo.com (M.F.A.E.-M.); hassanienmohamed@yahoo.com (M.F.R.) \\ 4 Agronomy Department, Faculty of Agriculture, Zagazig University, Zagazig 44519, Egypt \\ 5 Department of Biology, College of Science, Taif University, P.O. Box 11099, Taif 21944, Saudi Arabia; \\ m.fadl@tu.edu.sa \\ check for \\ * Correspondence: sayed_mansour_84@yahoo.es
} updates

Citation: Desoky, E.-S.M.; Merwad, A.-R.M.A.; Abo El-Maati, M.F.; Mansour, E.; Arnaout, S.M.A.I.; Awad, M.F; Ramadan, M.F.; Ibrahim, S.A. Physiological and Biochemical Mechanisms of Exogenously Applied Selenium for Alleviating Destructive Impacts Induced by Salinity Stress in Bread Wheat. Agronomy 2021, 11, 926. https://doi.org/10.3390/agronomy 11050926

Academic Editors: Mura Jyostna Devi and Leela Saisree Uppala

Received: 15 April 2021

Accepted: 4 May 2021

Published: 8 May 2021

Publisher's Note: MDPI stays neutral with regard to jurisdictional claims in published maps and institutional affiliations.

Copyright: () 2021 by the authors. Licensee MDPI, Basel, Switzerland. This article is an open access article distributed under the terms and conditions of the Creative Commons Attribution (CC BY) license (https:// creativecommons.org/licenses/by/ $4.0 /)$.
Abstract: Salinity is a major abiotic stress that poses great obstacles to wheat production, especially in arid regions. The application of exogenous substances can enhance plant salt tolerance and increase its productivity under salinity stress. This work aimed to assess the mechanisms of selenium (Se) at different concentrations $\left(2,4\right.$ and $\left.8 \mu \mathrm{M} \mathrm{SeCl}_{2}\right)$ to mitigate hazardous impacts of salt toxicity at physiological, biochemical and agronomic levels in bread wheat. The results displayed that Se foliar application increased chlorophyll content, net photosynthetic rate, transpiration rate, stomatal conductance, relative water content, membrane stability index, excised leaf water retention, proline, total soluble sugars, Ca content, $\mathrm{K}$ content, antioxidant enzyme activities and non-enzymatic antioxidant compounds compared to untreated plants. On the other hand, Se application decreased the content of $\mathrm{Na}$, hydrogen peroxide and superoxide contents. Accordingly, our findings recommend exogenous Se application (in particular $8 \mu \mathrm{M}$ ) to alleviate the deleterious effects induced by salinity stress and improve wheat yield attributes through enhancing antioxidant defense systems and photosynthetic capacity.

Keywords: salinity; wheat; selenium; physio-biochemical traits; growth; yield parameters

\section{Introduction}

Wheat (Triticum aestioum L.) belongs to the family Poaceae and is an important cereal crop worldwide. It is an essential grain source that provides humans with carbohydrates, vitamins, protein and extra calories. It is cultivated in almost 216 million hectares, producing approximately 766 million tons [1]. Its acreage in Egypt is about 1.41 million hectares, providing around 9.0 million tons [1]. Salinity is one of the main abiotic stresses that poses great constraints to agricultural production [2-4]. Various crops are responsive to salt stress and incapable of tolerating salinity even at low levels [5]. Irrigation using low-quality water is the major agent accumulating dissolved salt in the soil that inhibits the physiological process, metabolism and root-shoot growth and hence decreasing grain yield [6]. Plant metabolism can be modified through the toxicity of ions and osmotic stress resulting from using saline water for irrigation. Furthermore, salinity constrains plant growth by producing a high level of reactive oxygen species (ROS), such as hydrogen peroxide $\left(\mathrm{H}_{2} \mathrm{O}_{2}\right)$, superoxide $\left(\mathrm{O}_{2}^{-}\right)$and hydroxyl radicals $\left(\mathrm{OH}^{-}\right)$[7]. ROS is defined as 
the residue of normal cellular metabolism and is significant for the reaction of enzymatic intracellular coding when plants present salinity stress [8,9]. The grown plants under salinity suffer from toxic effects of $\mathrm{Na}^{+}$and $\mathrm{Cl}^{-}$ions and nutrient imbalance and diminish water availability in the soil [10]. Salt stress directly influences plant physiology throughout osmotic and ionic stress. It inhibits water relations of the plant, which leads to osmotic stress and water deficiency [6,11]. Salt accumulation in the leaf tissue causes dehydration and turgor, followed by the death of plant cells. Photosynthesis is extremely impacted by salt stress [12], which is joined by a decrease in $\mathrm{CO}_{2}$ pressure, stomata closure [13] and chlorophyll pigment $[11,14]$ and inhibition of rubisco and other biochemical operations, including activities of antioxidant enzymes $[15,16]$.

The current climate change fundamentally threatens the stability of the management and production of cereal crops. Accordingly, it is essential to find approaches to boost cereal growth and production, particularly under arid regions [17,18]. Selenium (Se) is an important microelement, but it is toxic in high concentrations to humans, animals, plants and microorganisms [19]. This toxicity is generated by replacing sulfur with Se in amino acids, which leads to faulty folding of the protein and, accordingly, nonfunctional proteins and enzymes. Se in small amounts can enhance plant tolerance to postpone senescence, oxidative stress, and stimulate plant growth $[20,21]$. The exogenous usage of Se at low concentrations mitigates salt-induced negative impacts [22,23]. Generally, it has been documented that it might play a vital role as an antioxidant involved in scavenging the dangerous impacts of free radicals [24-26]. Moreover, it was demonstrated that Se has a certain ability to enhance salt tolerance by promoting the plant growth $[27,28]$ aggregation of compatible solutes and photosynthetic pigments [22,28]. Accordingly, using Se foliar application could efficiently reduce the hazardous influences of salt toxicity on wheat plants. Nevertheless, the distinct alterations of physiological and biochemical processes in response to selenium under salinity stress remain to be elucidated. Consequently, the major objective of the current work was to assess the protective impact of Se foliar application on wheat's physio-biochemical properties, antioxidant defense system ingredients, growth and yield under salt conditions. Additionally, the study aimed to realize the mechanisms of salt tolerance in stressed plants that could be improved by Se application.

\section{Materials and Methods}

\subsection{Plant Materials and Experiment Design}

The used cultivar was Misr-1 (Triticum aestioum L.), which is a high-yielding commercial cultivar and is recommended by the Egyptian Ministry of Agriculture and Land Reclamation. Healthy seeds that were of good quality and free from insect infestations were carefully selected. The seeds were surface sterilized utilizing $1 \mathrm{~g} \mathrm{~kg}^{-1}$ mercury chloride $\left(\mathrm{HgCl}_{2}\right)$ for $1 \mathrm{~min}$; then, the seeds were washed with sterilized deionized water and air dried. Ten seeds were sown in each pot plastic ( $40 \mathrm{~cm}$ in depth and $35 \mathrm{~cm}$ in diameter) containing growth medium ion-free sand. At full emergence, only seven seedlings were kept. The experiment was conducted in a greenhouse during the two seasons of 2018-2019 and 2019-2020. The applied experimental design was a completely randomized design with ten replicates. The growing conditions in the two growing seasons were $55-58 \%$ humidity and $11 \pm 3 / 18 \pm 2{ }^{\circ} \mathrm{C}$ as night/day temperatures. Hoagland's nutrient solution ( $\frac{1}{2}$ strength) was used to irrigate the plants [29]. Every 5 days, the nutrient solution was applied at $100 \%$ field capacity, from sowing until full emergence, without any stress treatments to all pots. After full emergence ( 25 days after sowing), the pots were separated into three treatment groups: control (non-stressed), $200 \mathrm{mM} \mathrm{NaCl}$ (S1), and $250 \mathrm{mM} \mathrm{NaCl}$ (S2). The initial studies displayed that these levels had significant impacts on wheat seedling growth; consequently, they were selected. The applied concentrations of selenium $\left(\mathrm{SeCl}_{2}\right)$ were 2,4 and $8 \mu \mathrm{M}$. Spraying until dripping took place three times at 25, 40 and 55 days from sowing. Hand atomizer was used for spraying, and a few drops of Tween-20 (0.1\%) were utilized as a surfactant to ensure the penetration of solutions into the leaf tissue. Control treatment (without foliar spray) was sprayed with distilled water containing Tween-20. 
Soil $\mathrm{pH}$ was modified to the control $\mathrm{pH}(6.2-6.5)$ utilizing diluted sulfuric acid. Ten plants were taken after 65 days from sowing from each treatment to measure the dry weight of the shoot $\left(\mathrm{g} /\right.$ plant), plant height $(\mathrm{cm})$, leaf area $\left(\mathrm{cm}^{2}\right)$ and the physio-biochemical parameters. At physiological maturity (when the spikes had ripened and turned yellow), ten plants were randomly harvested to evaluate grain number/spike, 1000-grain weight (g) and grain yield/plant (g).

\subsection{Determination of Chlorophyll Content, PSII Quantum Yield and $\mathrm{CO}_{2}$ Fixation Rate}

Total carotenoids and chlorophyll contents were measured from fresh leaves of five plants using pure acetone as outlined by Fadeel [30]. Leaf net photosynthetic rate (Pn), stomatal conductance (gs) and rate of transpiration ( $\mathrm{Tr}$ ) were measured utilizing a portable photosynthesis system (LF6400XTR, LI-COR, USA); the measurements were taken between 09:00 and 11:00 a.m.

2.3. Determination of Relative Water Content (RWC), Membrane Stability Index (MSI), Excised Leaf Water Retention (ELWR), Leaf Soluble Sugars and Proline

RWC was determined as described by Barrs and Weatherley [31] MSI according to Premachandra et al. [32]; ELWR was estimated following Farshadfar et al. [33] using the following formula:

$$
\operatorname{ELWR}(\%)=\left[1-\frac{\mathrm{FW}-\mathrm{WW} 4 \mathrm{~h}}{\mathrm{FW}}\right] \times 100
$$

where FW is the fresh weight of leaves and WW4h is the wilted weight of leaves after $4 \mathrm{~h}$ at $25^{\circ} \mathrm{C}$. Total soluble sugar content was recorded as outlined by Irigoyen et al. [34]. The rapid colorimetric method was carried out to determine proline contents in $0.5 \mathrm{~g}$ dried leaf samples [35].

\subsection{Determination of Antioxidant Enzyme Activities}

Extraction from fresh leaves $(0.5 \mathrm{~g})$ was performed according to Mukherjee and Choudhuri [36]. The extract was frozen in liquid nitrogen and then ground in phosphate buffer $(100 \mathrm{mM}, \mathrm{pH} 7.0)$. Homogenates were centrifuged at $4{ }^{\circ} \mathrm{C}$ for $10 \mathrm{~min}$ under $15,000 \times g$. The supernatant was kept at $4{ }^{\circ} \mathrm{C}$ until used to measure the activity of peroxidase (POD), superoxide dismutase (SOD) and catalase (CAT). The nitro blue tetrazolium (NBT) test was used to analyze SOD (EC1.15.1.1) activity, defining its units as the enzyme amount required to inhibit 50\% of NBT as recorded at $560 \mathrm{~nm}$ [37]. An Aebi [38] test was applied to evaluate CAT (EC1.11.1.6) activity. The decline in absorbance recorded at $240 \mathrm{~nm}$ as an outcome of $\mathrm{H}_{2} \mathrm{O}_{2}$ consumption reveals the enzyme activity. The methods of Maechlay and Chance [39] and Klapheck et al. [40] were used to evaluate POD (EC1.11.1.7) activity. The rate of guaiacol oxidation in the presence of $\mathrm{H}_{2} \mathrm{O}_{2}$ recorded at $470 \mathrm{~nm}$ specifies the enzyme activity.

2.5. Determination of Non-Enzymatic Antioxidant Compounds and Oxidative Stress (Hydrogen Peroxide $\left(\mathrm{H}_{2} \mathrm{O}_{2}\right)$ and Superoxide $\left.\left(\mathrm{O}_{2}^{\bullet-}\right)\right)$

The content ( $\mu \mathrm{mol} \mathrm{g}{ }^{-1} \mathrm{FW}$ ) of ascorbate (AsA) was measured according to Kampfenkel et al. [41]. Contents ( $\mu \mathrm{mol} \mathrm{g}^{-1} \mathrm{FW}$ ) of reduced and total glutathione (GSH) were recorded according to Griffith [42]. Moreover, the methods of Konings et al. [43] and Ching and Mohamed [44] were used to determine $\alpha$-TOC. To measure $\mathrm{H}_{2} \mathrm{O}_{2}$ levels ( $\mu \mathrm{mol}$ per $\mathrm{g}$ of leaf FW), $0.25 \mathrm{~g}$ of fresh leaf was homogenized in $5 \mathrm{~mL}$ TCA (5\%). Absorbance reading was assessed at $390 \mathrm{~nm}$ using a standard prepared from $\mathrm{H}_{2} \mathrm{O}_{2}$ [45]. To measure $\mathrm{O}_{2}{ }^{\bullet-}$, wheat fresh leaf $(100 \mathrm{mg})$ was cut into $1 \mathrm{~mm} \times 1 \mathrm{~mm}$ fragments. Optical density was recorded at $580 \mathrm{~nm}$, and $\mathrm{O}_{2}{ }^{--}$content was expressed as $\mathrm{A} 580 \mathrm{~g}^{-1} \mathrm{FW}$ [46]. 


\subsection{Determinations of Potassium $\left(\mathrm{K}^{+}\right)$, Calcium $\left(\mathrm{Ca}^{2+}\right)$ and Sodium $\left(\mathrm{Na}^{+}\right)$}

Dried powdered leaves $(0.1 \mathrm{~g})$ were digested for $12 \mathrm{~h}$ using a mixture of perchloric acid $(2 \mathrm{~mL}, 80 \%)$ and concentrated $\mathrm{H}_{2} \mathrm{SO}_{4}(10 \mathrm{~mL})$. Each sample was diluted to $100 \mathrm{~mL}$ with distilled water to analyze $\mathrm{Ca}^{2+}, \mathrm{K}^{+}$and $\mathrm{Na}^{+}$contents using flame photometry [47].

\subsection{Statistical Analysis}

The data were analyzed using $\mathrm{R}$ software version 3.6.1. The least significant difference (LSD) at $p \leq 0.05$ was calculated to display the significant differences among investigated treatments.

\section{Results}

\subsection{Growth Parameters and Yield Components}

All measured agronomic traits of wheat were considerably diminished by salinity stress in the two growing seasons (Figures 1 and 2). Plant height, shoot dry weight, leaf area, number of grains/spike, 1000-grain weight and grain yield per plant significantly decreased on average by 16.6, 31.4, 26.3, 22.6, 4.1 and $34.0 \%$ under $200 \mathrm{mM} \mathrm{NaCl}$ and by $36.1,48.6,48.6,47.3,33.8$ and $67.9 \%$ under $250 \mathrm{mM} \mathrm{NaCl}$, respectively, compared with non-stressed treatment. The reductions in the growth traits were more marked under $250 \mathrm{mM}$ in comparison with $200 \mathrm{mM} \mathrm{NaCl}$. The application of Se significantly boosted all agronomic traits, especially at the concentration of $8 \mu \mathrm{M}$ Se when compared with 4 and $2 \mu \mathrm{M}$ Se. The application of $8 \mu \mathrm{M}$ Se enhanced plant height, shoot dry weight, leaf area, number of grains per spike, 1000-grain weight and grain yield/plant on average by $15.4,25.0,15.2,19.0,12.8$ and $41.2 \%$, respectively, compared untreated plants (sprayed with distilled water).

\subsection{Chlorophyll Content, Net Photosynthetic Rate (Pn), Transpiration Rate (Tr) and Stomatal Conductance ( $g s$ )}

It was observed that the $\mathrm{NaCl}$ salinity levels exhibited significant differences in chlorophyll content (total chlorophyll and total carotenoids), stomatal conductance (gs), net photosynthetic (Pn) and transpiration (Tr) rates in wheat during both growing seasons (Table 1). All aforementioned characteristics were considerably reduced by rising $\mathrm{NaCl}$ salinity levels to $200 \mathrm{mM} \mathrm{NaCl}$ by 12.9, 11.9, 25.8, 22.4 and $25.9 \%$, and by $26.8,20.8,46.6,39.9$ and $44.2 \%$, respectively, under $250 \mathrm{mM} \mathrm{NaCl}$, in the same order. Notwithstanding, foliar application with Se solution mitigated the harmful effects of salt stress and substantially elevated all measured characters. The greatest enhancement was observed for the Se dose at $8 \mu \mathrm{M}$ by $12.3,7.6,19.4,20.2$ and $11.3 \%$ compared with untreated plants, in the same order. 

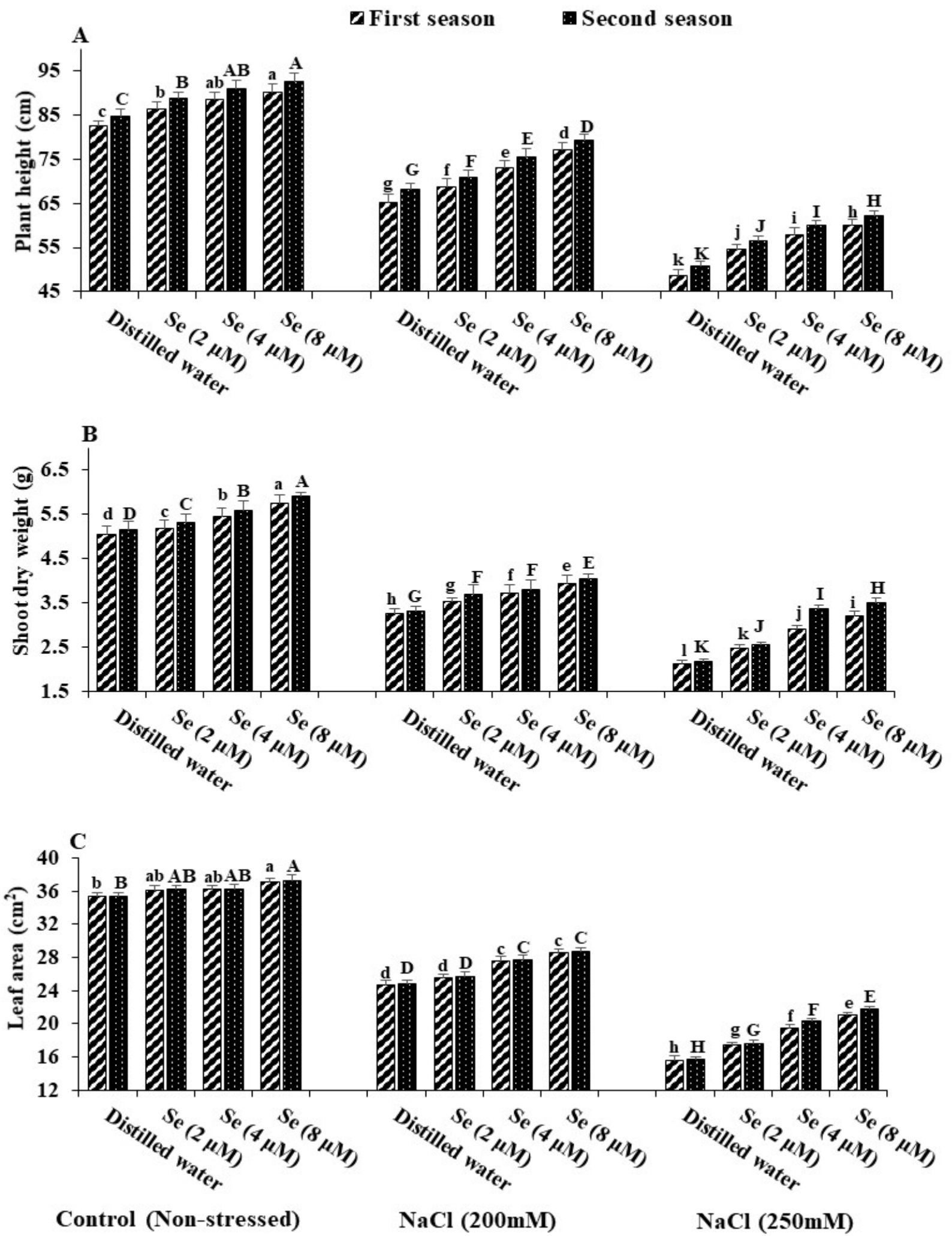

Figure 1. Impact of $\mathrm{NaCl}$ salinity stress and foliar spray with Se compared to distilled water on growth parameters of wheat plants; plant height (A), shoot dry weight (B) and leaf area (C) in the two growing seasons 2018-2019 (first season) and 2019-2020 (second season). The bars on the columns show the standard error of mean and different letters on the column differ significantly by LSD $(p<0.05)$, with uppercase letters belonging to the first season and lowercase letters belonging to the second season. 

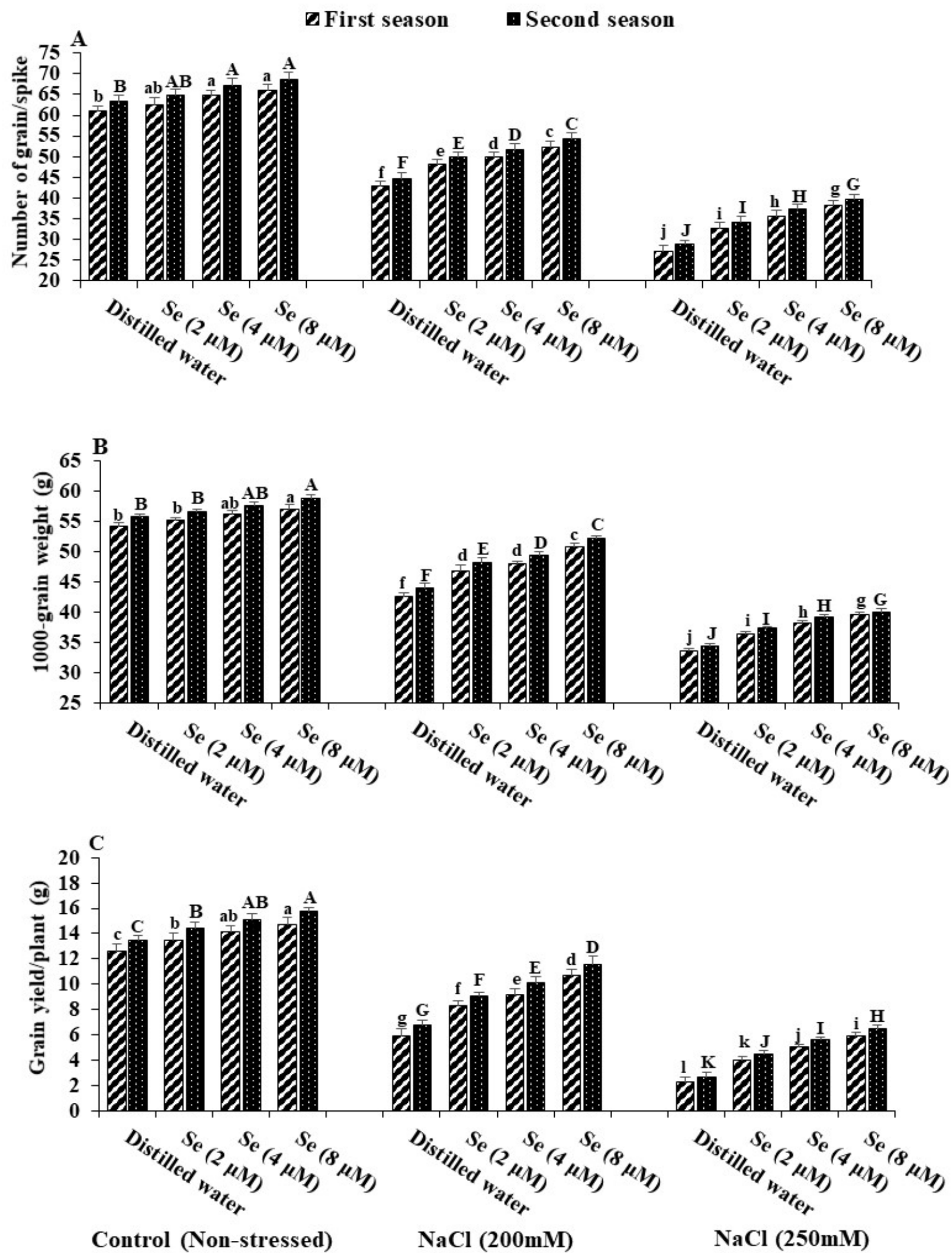

Figure 2. Impact of $\mathrm{NaCl}$ salinity stress and foliar spray with Se on yield components of wheat plants; number of grains per spike (A), 1000-grain weight (B) and grain yield per plant (C) in the two growing seasons 2018-2019 (first season) and 2019-2020 (second season). The bars on the columns show the standard error of mean and different letters on the column differ significantly by LSD $(p<0.05)$, with uppercase letters belonging to the first season and lowercase letters belonging to the second season. 
Table 1. Influence of $\mathrm{NaCl}$ salinity stress and foliar spray with Se on chlorophyll content, stomatal conductance (gs), net photosynthetic (Pn) and transpiration (Tr) rates and of wheat plants in the two seasons.

\begin{tabular}{|c|c|c|c|c|c|c|}
\hline Salinity & Foliar Spray & $\begin{array}{l}\text { Total } \\
\text { Chlorophylls } \\
\left(\mathrm{mg} \mathrm{g}^{-1} \mathrm{FW}\right)\end{array}$ & $\begin{array}{c}\text { Total } \\
\text { Carotenoids } \\
\left(\mathrm{mg} \mathrm{g}^{-1} \mathrm{FW}\right)\end{array}$ & $\begin{array}{c}\text { Net } \\
\text { Photosynthetic } \\
\text { Rate }\left(\mu \mathrm{mol} \mathrm{CO} \mathrm{CO}_{2}\right. \\
\left.\mathrm{m}^{-2} \mathrm{~s}^{-1}\right)\end{array}$ & $\begin{array}{c}\text { Transpiration } \\
\text { Rate }\left(\mathrm{mMol} \mathrm{H}_{2} \mathrm{O}\right. \\
\left.\mathrm{m}^{-2} \mathrm{~s}^{-1}\right)\end{array}$ & $\begin{array}{c}\text { Stomatal } \\
\text { Conductance } \\
\left(\mathrm{mMol}_{2} \mathrm{O}\right. \\
\left.\mathrm{m}^{-2} \mathrm{~s}^{-1}\right)\end{array}$ \\
\hline & & \multicolumn{5}{|c|}{ 1st Season } \\
\hline \multirow{5}{*}{ Control } & Distilled water & $2.22 \pm 0.05^{c}$ & $0.803 \pm 0.07^{c}$ & $10.3 \pm 0.6^{c}$ & $6.09 \pm 0.08^{\mathrm{d}}$ & $0.406 \pm 0.03^{\mathrm{d}}$ \\
\hline & Se $(2 \mu \mathrm{M})$ & $2.25 \pm 0.07^{b c}$ & $0.806 \pm 0.08^{b}$ & $11.2 \pm 0.7^{\mathrm{b}}$ & $6.23 \pm 0.09^{c}$ & $0.430 \pm 0.03^{c}$ \\
\hline & Se $(4 \mu \mathrm{M})$ & $2.29 \pm 0.05^{a b}$ & $0.826 \pm 0.06^{\mathrm{a}}$ & $11.5 \pm 0.9^{b}$ & $6.35 \pm 0.07^{b}$ & $0.450 \pm 0.02^{b}$ \\
\hline & Se $(8 \mu \mathrm{M})$ & $2.33 \pm 0.04^{a}$ & $0.836 \pm 0.06^{\mathrm{a}}$ & $14.7 \pm 0.8^{a}$ & $6.45 \pm 0.05^{\mathrm{a}}$ & $0.476 \pm 0.03^{a}$ \\
\hline & Distilled water & $1.85 \pm 0.02^{\mathrm{g}}$ & $0.693 \pm 0.04^{\mathrm{f}}$ & $8.26 \pm 0.4 \mathrm{~g}$ & $4.51 \pm 0.03^{h}$ & $0.296 \pm 0.01^{h}$ \\
\hline \multirow{3}{*}{$\mathrm{NaCl}(200 \mathrm{mM})$} & Se $(2 \mu \mathrm{M})$ & $1.95 \pm 0.03^{f}$ & $0.720 \pm 0.05^{\mathrm{e}}$ & $8.62 \pm 0.3^{f}$ & $4.67 \pm 0.02^{g}$ & $0.316 \pm 0.02^{g}$ \\
\hline & Se $(4 \mu \mathrm{M})$ & $2.01 \pm 0.04^{\mathrm{e}}$ & $0.733 \pm 0.06^{\mathrm{d}}$ & $9.04 \pm 0.7^{\mathrm{e}}$ & $4.79 \pm 0.03^{f}$ & $0.336 \pm 0.02^{f}$ \\
\hline & Se $(8 \mu \mathrm{M})$ & $2.14 \pm 0.03^{\mathrm{d}}$ & $0.750 \pm 0.06^{c}$ & $9.59 \pm 0.7^{\mathrm{d}}$ & $4.94 \pm 0.03^{\mathrm{e}}$ & $0.356 \pm 0.03^{\mathrm{e}}$ \\
\hline \multirow{5}{*}{$\mathrm{NaCl}(250 \mathrm{mM})$} & Distilled water & $1.52 \pm 0.01^{j}$ & $0.610 \pm 0.04^{\mathrm{i}}$ & $6.21 \pm 0.3^{\mathrm{k}}$ & $3.21 \pm 0.02^{1}$ & $0.213 \pm 0.01^{1}$ \\
\hline & Se $(2 \mu \mathrm{M})$ & $1.62 \pm 0.03^{i}$ & $0.640 \pm 0.03^{h}$ & $6.65 \pm 0.5^{j}$ & $3.51 \pm 0.02^{k}$ & $0.236 \pm 0.01^{k}$ \\
\hline & Se $(4 \mu \mathrm{M})$ & $1.76 \pm 0.02^{h}$ & $0.666 \pm 0.02 \mathrm{~g}$ & $7.11 \pm 0.2^{\mathrm{hi}}$ & $3.79 \pm 0.03^{j}$ & $0.260 \pm 0.01^{j}$ \\
\hline & Se $(8 \mu \mathrm{M})$ & $1.81 \pm 0.04^{g}$ & $0.683 \pm 0.03^{f}$ & $7.70 \pm 0.3^{h}$ & $4.02 \pm 0.05^{\mathrm{i}}$ & $0.276 \pm 0.01^{\mathrm{i}}$ \\
\hline & & \multicolumn{5}{|c|}{ 2nd Season } \\
\hline \multirow{5}{*}{ Control } & Distilled water & $2.30 \pm 0.06^{c}$ & $0.833 \pm 0.08^{b}$ & $11.1 \pm 0.5^{c}$ & $6.69 \pm 0.10^{d}$ & $0.486 \pm 0.03^{d}$ \\
\hline & Se $(2 \mu \mathrm{M})$ & $2.33 \pm 0.07^{b c}$ & $0.836 \pm 0.09^{b}$ & $12.0 \pm 0.8^{b}$ & $6.83 \pm 0.09^{c}$ & $0.510 \pm 0.04^{c}$ \\
\hline & Se $(4 \mu \mathrm{M})$ & $2.37 \pm 0.06^{\mathrm{ab}}$ & $0.856 \pm 0.08^{a}$ & $12.3 \pm 1.0^{b}$ & $6.95 \pm 0.09^{b}$ & $0.530 \pm 0.04^{b}$ \\
\hline & Se $(8 \mu \mathrm{M})$ & $2.42 \pm 0.07^{\mathrm{a}}$ & $0.866 \pm 0.07^{a}$ & $13.5 \pm 1.1^{\mathrm{a}}$ & $7.05 \pm 0.10^{\mathrm{a}}$ & $0.556 \pm 0.03^{\mathrm{a}}$ \\
\hline & Distilled water & $1.90 \pm 0.09 \mathrm{~g}$ & $0.713 \pm 0.06^{\mathrm{f}}$ & $8.86 \pm 0.6^{g}$ & $4.81 \pm 0.09^{h}$ & $0.356 \pm 0.02^{h}$ \\
\hline \multirow{3}{*}{$\mathrm{NaCl}(200 \mathrm{mM})$} & Se $(2 \mu \mathrm{M})$ & $2.01 \pm 0.02^{\mathrm{f}}$ & $0.740 \pm 0.04^{\mathrm{e}}$ & $9.22 \pm 0.8^{\mathrm{f}}$ & $4.97 \pm 0.07 \mathrm{~g}$ & $0.376 \pm 0.02 \mathrm{~g}$ \\
\hline & Se $(4 \mu \mathrm{M})$ & $2.06 \pm 0.03^{\mathrm{e}}$ & $0.753 \pm 0.03^{\mathrm{d}}$ & $9.64 \pm 0.9^{\mathrm{e}}$ & $5.09 \pm 0.06^{f}$ & $0.396 \pm 0.01^{\mathrm{e}}$ \\
\hline & Se $(8 \mu \mathrm{M})$ & $2.20 \pm 0.01^{\mathrm{d}}$ & $0.770 \pm 0.04^{c}$ & $10.1 \pm 0.8^{\mathrm{d}}$ & $5.24 \pm 0.03^{\mathrm{e}}$ & $0.416 \pm 0.02^{\mathrm{e}}$ \\
\hline \multirow{4}{*}{$\mathrm{NaCl}(250 \mathrm{mM})$} & Distilled water & $1.55 \pm 0.02^{k}$ & $0.630 \pm 0.04^{i}$ & $6.61 \pm 0.5^{\mathrm{k}}$ & $3.29 \pm 0.06^{1}$ & $0.233 \pm 0.01^{1}$ \\
\hline & Se $(2 \mu \mathrm{M})$ & $1.65 \pm 0.03^{j}$ & $0.660 \pm 0.02^{h}$ & $7.05 \pm 0.2^{j}$ & $3.59 \pm 0.07^{\mathrm{k}}$ & $0.256 \pm 0.01^{\mathrm{k}}$ \\
\hline & Se $(4 \mu \mathrm{M})$ & $1.79 \pm 0.02^{\mathrm{i}}$ & $0.686 \pm 0.03 \mathrm{~g}$ & $7.51 \pm 0.3^{i}$ & $3.87 \pm 0.05^{j}$ & $0.280 \pm 0.02^{j}$ \\
\hline & Se $(8 \mu \mathrm{M})$ & $1.84 \pm 0.04^{h}$ & $0.703 \pm 0.03^{f}$ & $8.10 \pm 0.4^{h}$ & $4.10 \pm 0.08^{i}$ & $0.296 \pm 0.01^{\mathrm{i}}$ \\
\hline
\end{tabular}

Means followed by the same letters are not significantly different by LSD $(p<0.05)$.

\subsection{Relative Water Content (RWC), Membrane Stability Index (MSI), Excised Leaf Water Retention (ELWR), Proline and Total Soluble Sugars}

The RWC, MSI and ELWR of wheat leaves were considerably diminished by increasing $\mathrm{NaCl}$ salinity stress. RWC, MSI, and ELWR decreased by $12.5,21.2$ and $10.8 \%$ under $200 \mathrm{mM}$ $\mathrm{NaCl}$, and by $21.9,42.5$ and $25.5 \%$ under $250 \mathrm{mM} \mathrm{NaCl}$, respectively. On the contrary, proline content and total soluble sugars were positively associated with rising salinity levels in both seasons (Table 2). The exogenous Se application on wheat plants displayed a considerable increase in RWC, MSI, ELWR, proline content and total soluble sugars under unstressed and stressed conditions. Furthermore, Se foliar spray effectively reduced the undesirable impact of salinity on RWC, MSI, ELWR, proline content and total soluble sugars. In addition, the highest dose of Se $(8 \mu \mathrm{M})$ was more effective in mitigating the deleterious effect of salinity stress and enhanced RWC, MSI, ELWR, proline content and total soluble sugars by $7.4,15.9,5.2,15.4$ and $4.8 \%$, respectively, in comparison with untreated plants (sprayed with distilled water) over the two growing seasons. 
Table 2. Influence of $\mathrm{NaCl}$ salinity stress and foliar spray with Se on relative water content (RWC), membrane stability index (MSI), excised leaf water retention (ELWR), proline and total soluble sugars of wheat plants in the two seasons.

\begin{tabular}{|c|c|c|c|c|c|c|}
\hline Salinity & Foliar Spray & $\begin{array}{c}\text { RWC } \\
(\%)\end{array}$ & $\begin{array}{l}\text { MSI } \\
(\%)\end{array}$ & $\begin{array}{c}\text { ELWR } \\
(\%)\end{array}$ & $\begin{array}{c}\text { Free Proline } \\
\left(\mu \mathrm{g}^{-1} \text { Dry Weight) }\right.\end{array}$ & $\begin{array}{l}\text { Total Soluble Sugars } \\
\text { (mg g-1 Dry Weight) }\end{array}$ \\
\hline & & \multicolumn{5}{|c|}{ 1st Season } \\
\hline \multirow{5}{*}{ Control } & Distilled water & $71.3 \pm 1.1^{\mathrm{d}}$ & $52.5 \pm 0.8^{\mathrm{d}}$ & $75.5 \pm 0.5^{\mathrm{d}}$ & $65.2 \pm 0.4^{j}$ & $20.6 \pm 0.3^{k}$ \\
\hline & Se $(2 \mu \mathrm{M})$ & $73.4 \pm 1.0^{c}$ & $57.1 \pm 0.6^{c}$ & $76.7 \pm 0.7^{c}$ & $66.7 \pm 0.6^{\mathrm{ij}}$ & $21.0 \pm 0.2^{\mathrm{jk}}$ \\
\hline & Se $(4 \mu \mathrm{M})$ & $74.6 \pm 1.3^{b}$ & $58.4 \pm 0.7^{\mathrm{b}}$ & $77.8 \pm 0.8^{b}$ & $67.1 \pm 0.4^{\mathrm{ij}}$ & $21.8 \pm 0.2^{\mathrm{ij}}$ \\
\hline & Se $(8 \mu \mathrm{M})$ & $75.7 \pm 1.2^{\mathrm{a}}$ & $60.2 \pm 0.4^{\mathrm{a}}$ & $78.5 \pm 0.5^{\mathrm{a}}$ & $67.8 \pm 0.3^{i}$ & $22.5 \pm 0.3^{i}$ \\
\hline & Distilled water & $62.0 \pm 0.9^{\mathrm{h}}$ & $40.5 \pm 0.8^{h}$ & $67.5 \pm 0.3^{h}$ & $141.1 \pm 0.9^{\mathrm{h}}$ & $36.9 \pm 0.5^{h}$ \\
\hline \multirow{3}{*}{$\mathrm{NaCl}(200 \mathrm{mM})$} & Se $(2 \mu \mathrm{M})$ & $63.6 \pm 0.9 \mathrm{~g}$ & $43.9 \pm 0.4 \mathrm{~g}$ & $68.4 \pm 0.6^{g}$ & $146.0 \pm 0.8^{g}$ & $39.3 \pm 0.5 \mathrm{~g}$ \\
\hline & Se $(4 \mu \mathrm{M})$ & $65.3 \pm 1.1^{\mathrm{f}}$ & $46.6 \pm 0.6^{\mathrm{f}}$ & $69.4 \pm 0.4^{\mathrm{f}}$ & $153.6 \pm 0.9^{\mathrm{f}}$ & $41.9 \pm 0.7^{\mathrm{f}}$ \\
\hline & Se $(8 \mu \mathrm{M})$ & $67.3 \pm 0.9 \mathrm{e}$ & $50.4 \pm 0.9^{\mathrm{e}}$ & $70.4 \pm 0.9^{\mathrm{e}}$ & $159.0 \pm 1.0^{\mathrm{e}}$ & $44.8 \pm 0.9^{\mathrm{e}}$ \\
\hline \multirow{4}{*}{$\mathrm{NaCl}(250 \mathrm{mM})$} & Distilled water & $55.7 \pm 0.7^{1}$ & $30.6 \pm 0.4^{1}$ & $55.2 \pm 0.8^{1}$ & $173.0 \pm 1.1^{\mathrm{d}}$ & $48.7 \pm 0.8^{\mathrm{d}}$ \\
\hline & Se $(2 \mu \mathrm{M})$ & $65.7 \pm 0.9^{\mathrm{k}}$ & $32.3 \pm 0.5^{\mathrm{k}}$ & $57.1 \pm 0.8^{\mathrm{k}}$ & $176.0 \pm 0.8^{c}$ & $50.6 \pm 0.9^{c}$ \\
\hline & Se $(4 \mu \mathrm{M})$ & $58.1 \pm 0.8^{j}$ & $34.1 \pm 0.6^{j}$ & $58.7 \pm 0.4^{j}$ & $178.6 \pm 0.9^{b}$ & $53.2 \pm 0.7^{b}$ \\
\hline & Se $(8 \mu \mathrm{M})$ & $59.9 \pm 0.6^{\mathrm{i}}$ & $36.1 \pm 0.4^{\mathrm{i}}$ & $59.9 \pm 0.5^{\mathrm{i}}$ & $182.4 \pm 0.9^{a}$ & $55.9 \pm 0.8^{a}$ \\
\hline & & \multicolumn{5}{|c|}{ 2nd Season } \\
\hline \multirow{5}{*}{ Control } & Distilled water & $72.6 \pm 0.9^{d}$ & $56.2 \pm 0.6^{\mathrm{d}}$ & $77.7 \pm 0.9^{d}$ & $67.1 \pm 0.5^{j}$ & $22.3 \pm 0.2^{\mathrm{k}}$ \\
\hline & Se $(2 \mu \mathrm{M})$ & $74.7 \pm 0.8^{c}$ & $58.0 \pm 0.9^{c}$ & $78.8 \pm 0.6^{c}$ & $69.4 \pm 0.6^{\mathrm{i}}$ & $22.6 \pm 0.4^{\mathrm{jk}}$ \\
\hline & Se $(4 \mu \mathrm{M})$ & $76.0 \pm 0.9^{b}$ & $59.4 \pm 0.7^{b}$ & $79.9 \pm 0.5^{b}$ & $69.7 \pm 0.3^{\mathrm{i}}$ & $23.5 \pm 0.2^{\mathrm{ij}}$ \\
\hline & Se $(8 \mu \mathrm{M})$ & $77.1 \pm 0.5^{\mathrm{a}}$ & $61.2 \pm 0.8^{\mathrm{a}}$ & $80.6 \pm 0.5^{\mathrm{a}}$ & $70.4 \pm 0.6^{\mathrm{i}}$ & $24.1 \pm 0.5^{\mathrm{i}}$ \\
\hline & Distilled water & $63.2 \pm 0.7^{\mathrm{h}}$ & $41.5 \pm 0.5^{h}$ & $69.1 \pm 0.8^{h}$ & $145.6 \pm 0.9^{h}$ & $39.5 \pm 0.3^{h}$ \\
\hline \multirow{3}{*}{$\mathrm{NaCl}(200 \mathrm{mM})$} & Se $(2 \mu \mathrm{M})$ & $64.8 \pm 0.4^{g}$ & $44.8 \pm 0.7^{g}$ & $70.2 \pm 0.6^{g}$ & $150.5 \pm 0.8^{g}$ & $42.0 \pm 0.4 \mathrm{~g}$ \\
\hline & Se $(4 \mu \mathrm{M})$ & $66.5 \pm 0.7^{f}$ & $47.6 \pm 0.6^{\mathrm{f}}$ & $71.0 \pm 0.8^{\mathrm{f}}$ & $158.3 \pm 0.9^{\mathrm{f}}$ & $44.5 \pm 0.2^{\mathrm{f}}$ \\
\hline & Se $(8 \mu \mathrm{M})$ & $68.6 \pm 0.9^{\mathrm{e}}$ & $51.3 \pm 0.7^{\mathrm{e}}$ & $72.0 \pm 0.4^{\mathrm{e}}$ & $163.6 \pm 0.8^{\mathrm{e}}$ & $47.4 \pm 0.6^{\mathrm{e}}$ \\
\hline \multirow{4}{*}{$\mathrm{NaCl}(250 \mathrm{mM})$} & Distilled water & $56.8 \pm 0.5^{1}$ & $30.9 \pm 0.5^{1}$ & $56.2 \pm 0.4^{1}$ & $179.9 \pm 0.9^{\mathrm{d}}$ & $52.6 \pm 0.8^{d}$ \\
\hline & Se $(2 \mu \mathrm{M})$ & $57.8 \pm 0.8^{\mathrm{k}}$ & $32.6 \pm 0.4^{\mathrm{k}}$ & $58.1 \pm 0.9^{\mathrm{k}}$ & $182.9 \pm 0.6^{c}$ & $54.5 \pm 0.7^{c}$ \\
\hline & Se $(4 \mu \mathrm{M})$ & $59.3 \pm 0.6^{j}$ & $34.5 \pm 0.5^{\mathrm{j}}$ & $59.7 \pm 0.5^{j}$ & $185.6 \pm 1.0^{\mathrm{b}}$ & $57.2 \pm 0.6^{b}$ \\
\hline & Se $(8 \mu \mathrm{M})$ & $61.1 \pm 0.6^{\mathrm{i}}$ & $36.4 \pm 0.4^{\mathrm{i}}$ & $60.9 \pm 0.4^{\mathrm{i}}$ & $189.4 \pm 0.9^{\mathrm{a}}$ & $59.8 \pm 0.8^{a}$ \\
\hline
\end{tabular}

Means followed by the same letters are not significantly different by LSD $(p<0.05)$.

\subsection{Antioxidant Enzyme (POX, CAT and SOD) Activities and Non-Enzymatic Antioxidants (AsA, GSH and $\alpha-T O C$ )}

Under salinity stress, antioxidant enzyme (POX, CAT and SOD) activities and nonenzymatic antioxidants (AsA, GSH and $\alpha$-TOC) in wheat plants were superior in comparison with normal growth conditions during the two growing seasons (Table 3). Likewise, Se foliar spray significantly enhanced the antioxidant enzyme activities and non-enzymatic antioxidants compared to distilled water application. Moreover, the maximum enhancement of antioxidant enzymes (POX, CAT and SOD) and non-enzymatic antioxidant compounds (AsA, GSH and $\alpha$-TOC) was recorded for foliar spray at $8 \mu \mathrm{M}$ as $6.6,9.0,7.4,10.8,4.6$ and $15.0 \%$, respectively. The increase in antioxidant enzyme activities and non-enzymatic antioxidant compounds assisted wheat plants to alleviate the negative effects of $\mathrm{NaCl}$ salinity stress. 
Table 3. Influence of $\mathrm{NaCl}$ salinity stress and foliar spray with Se on antioxidant enzyme activities and non-enzymatic antioxidant compounds of wheat plants in the two seasons.

\begin{tabular}{|c|c|c|c|c|c|c|c|}
\hline \multirow[b]{2}{*}{ Salinity } & \multirow[b]{2}{*}{ Foliar Spray } & \multicolumn{3}{|c|}{ Antioxidant Enzymes } & \multicolumn{3}{|c|}{ Non-Enzymatic Antioxidant } \\
\hline & & $\begin{array}{c}\text { CAT } \\
\left(\mathrm{U} \mathrm{mg}^{-1}\right. \\
\left.\text { min }^{-1}\right)\end{array}$ & $\begin{array}{c}\text { POX } \\
\left(\mu \mathrm{g} \mathrm{g}^{-1} \text { fresh }\right. \\
\left.\text { weight } \text { min }^{-1}\right)\end{array}$ & $\begin{array}{c}\text { SOD } \\
\text { (U } \mu g^{-1} \\
\text { protein) }\end{array}$ & $\begin{array}{c}\text { AsA } \\
\left(\mu \mathrm{mol} \mathrm{g}{ }^{-1} \mathrm{FW}\right)\end{array}$ & $\begin{array}{c}\text { GsH } \\
\left(\mu \mathrm{mol} \mathrm{g}{ }^{-1} \text { FW }\right)\end{array}$ & $\begin{array}{c}\alpha-\mathrm{TOC} \\
\left(\mu \mathrm{mol} \mathrm{g}{ }^{-1}\right. \\
\text { DW) }\end{array}$ \\
\hline & & \multicolumn{6}{|c|}{ 1st Season } \\
\hline \multirow{5}{*}{ Control } & Distilled water & $0.436 \pm 0.003^{1}$ & $75.9 \pm 0.6^{\mathrm{k}}$ & $40.2 \pm 0.2^{j}$ & $1.21 \pm 0.009^{k}$ & $0.312 \pm 0.001^{1}$ & $1.36 \pm 0.02^{1}$ \\
\hline & Se $(2 \mu \mathrm{M})$ & $0.453 \pm 0.0002^{k}$ & $77.0 \pm 0.9^{\mathrm{jk}}$ & $40.8 \pm 0.1^{\mathrm{ij}}$ & $1.26 \pm 0.005^{j}$ & $0.316 \pm 0.001^{k}$ & $1.43 \pm 0.03^{k}$ \\
\hline & Se $(4 \mu \mathrm{M})$ & $0.466 \pm 0.004^{j}$ & $77.9 \pm 0.7^{\mathrm{ij}}$ & $41.9 \pm 0.3^{\mathrm{hi}}$ & $1.30 \pm 0.006^{\mathrm{i}}$ & $0.322 \pm 0.001^{j}$ & $1.51 \pm 0.01^{\mathrm{j}}$ \\
\hline & Se $(8 \mu \mathrm{M})$ & $0.486 \pm 0.002^{i}$ & $78.7 \pm 0.9^{\mathrm{i}}$ & $42.7 \pm 0.4^{\mathrm{h}}$ & $1.37 \pm 0.005^{\mathrm{h}}$ & $0.329 \pm 0.002^{\mathrm{i}}$ & $1.57 \pm 0.02^{\mathrm{i}}$ \\
\hline & Distilled water & $0.706 \pm 0.006^{\mathrm{h}}$ & $115.9 \pm 0.8^{h}$ & $61.1 \pm 0.8^{\mathrm{g}}$ & $2.11 \pm 0.009 \mathrm{~g}$ & $0.443 \pm 0.001^{h}$ & $2.16 \pm 0.05^{\mathrm{h}}$ \\
\hline \multirow{3}{*}{$\mathrm{NaCl}(200 \mathrm{mM})$} & Se $(2 \mu \mathrm{M})$ & $0.726 \pm 0.005^{g}$ & $120.6 \pm 0.6^{g}$ & $62.9 \pm 0.9^{\mathrm{f}}$ & $2.21 \pm 0.005^{\mathrm{f}}$ & $0.449 \pm 0.002 \mathrm{~g}$ & $2.25 \pm 0.06^{\mathrm{g}}$ \\
\hline & Se $(4 \mu \mathrm{M})$ & $0.740 \pm 0.004^{\mathrm{f}}$ & $123.3 \pm 0.6^{\mathrm{f}}$ & $63.8 \pm 0.7^{f}$ & $2.28 \pm 0.006^{\mathrm{e}}$ & $0.453 \pm 0.003^{f}$ & $2.38 \pm 0.07^{\mathrm{f}}$ \\
\hline & Se $(8 \mu \mathrm{M})$ & $0.763 \pm 0.005^{\mathrm{e}}$ & $126.9 \pm 0.8^{\mathrm{e}}$ & $65.1 \pm 0.6^{\mathrm{e}}$ & $2.37 \pm 0.008^{\mathrm{d}}$ & $0.459 \pm 0.002^{\mathrm{e}}$ & $2.63 \pm 0.05^{\mathrm{e}}$ \\
\hline \multirow{5}{*}{$\mathrm{NaCl}(250 \mathrm{mM})$} & Distilled water & $0.816 \pm 0.006^{\mathrm{d}}$ & $133.2 \pm 0.7^{\mathrm{d}}$ & $70.8 \pm 0.5^{\mathrm{d}}$ & $2.41 \pm 0.007^{\mathrm{d}}$ & $0.462 \pm 0.001^{\mathrm{d}}$ & $3.28 \pm 0.06^{\mathrm{d}}$ \\
\hline & Se $(2 \mu \mathrm{M})$ & $0.843 \pm 0.003^{c}$ & $136.6 \pm 0.9^{c}$ & $72.8 \pm 0.7^{c}$ & $2.46 \pm 0.006^{c}$ & $0.465 \pm 0.003^{c}$ & $3.39 \pm 0.07^{c}$ \\
\hline & Se $(4 \mu \mathrm{M})$ & $0.870 \pm 0.005^{b}$ & $138.8 \pm 0.6^{b}$ & $74.8 \pm 0.6^{b}$ & $2.52 \pm 0.008^{b}$ & $0.469 \pm 0.002^{b}$ & $3.47 \pm 0.05^{b}$ \\
\hline & Se $(8 \mu \mathrm{M})$ & $0.893 \pm 0.006^{\mathrm{a}}$ & $141.2 \pm 0.8^{\mathrm{a}}$ & $77.3 \pm 0.3^{a}$ & $2.59 \pm 0.009^{a}$ & $0.478 \pm 0.003^{\mathrm{a}}$ & $3.60 \pm 0.06^{\mathrm{a}}$ \\
\hline & & \multicolumn{6}{|c|}{ 2nd Season } \\
\hline \multirow{5}{*}{ Control } & Distilled water & $0.466 \pm 0.002^{1}$ & $77.9 \pm 0.5^{k}$ & $41.8 \pm 0.4^{j}$ & $1.23 \pm 0.006^{\mathrm{k}}$ & $0.332 \pm 0.001^{k}$ & $1.32 \pm 0.03^{1}$ \\
\hline & Se $(2 \mu \mathrm{M})$ & $0.483 \pm 0.003^{\mathrm{k}}$ & $79.0 \pm 0.4^{j \mathrm{k}}$ & $42.4 \pm 0.2^{\mathrm{ij}}$ & $1.28 \pm 0.003^{\mathrm{j}}$ & $0.336 \pm 0.001^{j}$ & $1.39 \pm 0.02^{\mathrm{k}}$ \\
\hline & Se $(4 \mu \mathrm{M})$ & $0.497 \pm 0.002^{j}$ & $80.0 \pm 0.4{ }^{i j}$ & $43.5 \pm 0.1 \mathrm{hi}$ & $1.33 \pm 0.005^{\mathrm{i}}$ & $0.352 \pm 0.002^{\mathrm{i}}$ & $1.47 \pm 0.05^{\mathrm{j}}$ \\
\hline & Se $(8 \mu \mathrm{M})$ & $0.516 \pm 0.004^{\mathrm{i}}$ & $80.7 \pm 0.5^{\mathrm{i}}$ & $44.3 \pm 0.4^{\mathrm{h}}$ & $1.40 \pm 0.003^{h}$ & $0.359 \pm 0.002^{h}$ & $1.53 \pm 0.03^{\mathrm{i}}$ \\
\hline & Distilled water & $0.756 \pm 0.005^{h}$ & $120.0 \pm 0.6^{\mathrm{h}}$ & $63.5 \pm 0.6^{\mathrm{g}}$ & $2.15 \pm 0.005^{g}$ & $0.483 \pm 0.002 \mathrm{~g}$ & $2.10 \pm 0.06^{\mathrm{h}}$ \\
\hline \multirow{3}{*}{$\mathrm{NaCl}(200 \mathrm{mM})$} & Se $(2 \mu \mathrm{M})$ & $0.776 \pm 0.004^{g}$ & $124.6 \pm 0.8^{g}$ & $65.3 \pm 0.7^{f}$ & $2.25 \pm 0.007^{\mathrm{f}}$ & $0.489 \pm 0.003^{f}$ & $2.19 \pm 0.07^{g}$ \\
\hline & Se $(4 \mu \mathrm{M})$ & $0.790 \pm 0.003^{f}$ & $127.4 \pm 0.6^{\mathrm{f}}$ & $66.2 \pm 0.2^{f}$ & $2.32 \pm 0.009 \mathrm{e}$ & $0.496 \pm 0.001^{\mathrm{e}}$ & $2.32 \pm 0.07^{f}$ \\
\hline & Se $(8 \mu \mathrm{M})$ & $0.813 \pm 0.006^{\mathrm{e}}$ & $130.9 \pm 0.8^{\mathrm{e}}$ & $67.5 \pm 0.9^{\mathrm{e}}$ & $2.42 \pm 0.006^{\mathrm{d}}$ & $0.509 \pm 0.002^{d}$ & $2.57 \pm 0.06^{\mathrm{e}}$ \\
\hline \multirow{4}{*}{$\mathrm{NaCl}(250 \mathrm{mM})$} & Distilled water & $0.876 \pm 0.003^{d}$ & $138.7 \pm 0.8^{\mathrm{d}}$ & $74.6 \pm 0.8^{\mathrm{d}}$ & $2.46 \pm 0.005^{\mathrm{d}}$ & $0.512 \pm 0.003^{d}$ & $3.19 \pm 0.09^{d}$ \\
\hline & Se $(2 \mu \mathrm{M})$ & $0.903 \pm 0.006^{c}$ & $142.1 \pm 0.5^{c}$ & $76.7 \pm 0.5^{c}$ & $2.51 \pm 0.007^{c}$ & $0.515 \pm 0.005^{c}$ & $3.30 \pm 0.07^{c}$ \\
\hline & Se $(4 \mu \mathrm{M})$ & $0.930 \pm 0.007^{b}$ & $144.3 \pm 0.7^{b}$ & $78.6 \pm 0.5^{b}$ & $2.57 \pm 0.004^{b}$ & $0.519 \pm 0.003^{b}$ & $3.38 \pm 0.08^{b}$ \\
\hline & Se $(8 \mu \mathrm{M})$ & $0.953 \pm 0.007^{\mathrm{a}}$ & $146.7 \pm 0.9^{\mathrm{a}}$ & $81.2 \pm 0.6^{\mathrm{a}}$ & $2.64 \pm 0.005^{\mathrm{a}}$ & $0.528 \pm 0.004^{\mathrm{a}}$ & $3.51 \pm 0.09^{\mathrm{a}}$ \\
\hline
\end{tabular}

Means followed by the same letters are not significantly different by LSD $(p<0.05)$.

\subsection{Mineral ( $\mathrm{Ca}, \mathrm{K}$ and $\mathrm{Na}$ ) Uptake, $\mathrm{K}^{+} / \mathrm{Na}^{+}$Ratio and Oxidative Stress $\left(\mathrm{H}_{2} \mathrm{O}_{2}\right.$ and $\left.\mathrm{O}_{2}{ }^{\bullet-}\right)$}

The mineral nutrients uptake, the $\mathrm{K}^{+} / \mathrm{Na}^{+}$ratio and oxidative stress in wheat plants induced by salinity stress and treated by Se foliar spray are presented in Table 4 . It can be clearly seen that $\mathrm{NaCl}$ salinity stress and foliar application interacted and caused significant changes to all of the above-mentioned attributes. $\mathrm{Na}, \mathrm{H}_{2} \mathrm{O}_{2}$ and $\mathrm{O}_{2}{ }^{\bullet-}$ contents were significantly increased in wheat plants under $200 \mathrm{mM} \mathrm{NaCl}$ by $369,25.9$ and $76.5 \%$, and by $560,50.9$ and $178.8 \%$ under $250 \mathrm{mM} \mathrm{NaCl}$, respectively. Moreover, $\mathrm{Ca}$ and $\mathrm{K}$ content and the $\mathrm{K}^{+} / \mathrm{Na}^{+}$ratio were considerably diminished by $31.2,27.2,84.3 \%$ under $200 \mathrm{mM} \mathrm{NaCl}$, and by 44.7, 45.2 and $91.5 \%$ under $250 \mathrm{mM} \mathrm{NaCl}$, respectively. Nevertheless, the sprayed plants by Se had considerably lower values of $\mathrm{Na}, \mathrm{H}_{2} \mathrm{O}_{2}$ and $\mathrm{O}_{2}{ }^{\bullet-}$ contents compared to untreated ones under $\mathrm{NaCl}$ salinity stress conditions. Furthermore, exogenous Se application enhanced $\mathrm{Ca}$ and $\mathrm{K}$ contents and the $\mathrm{K}^{+} / \mathrm{Na}^{+}$ratio in comparison with untreated plants in both growing seasons. 
Table 4. Influence of $\mathrm{NaCl}$ salinity stress and foliar spray with Se on mineral uptake content, the $\mathrm{K}^{+} / \mathrm{Na}^{+}$ratio and oxidative stress of wheat plants in the two seasons.

\begin{tabular}{|c|c|c|c|c|c|c|c|}
\hline \multirow[b]{2}{*}{ Salinity } & \multirow[b]{2}{*}{ Foliar Spray } & \multicolumn{3}{|c|}{ Mineral Uptake (mg g ${ }^{-1}$ Dry Weight) } & \multirow[b]{2}{*}{$\mathrm{K}^{+} / \mathrm{Na}^{+}$ratio } & \multicolumn{2}{|c|}{ Oxidative Stress } \\
\hline & & Ca & $\mathbf{K}$ & $\mathrm{Na}$ & & $\begin{array}{c}\mathrm{O}_{2}{ }^{\bullet-} \\
\left(\mathrm{A} 580 \mathrm{~g}^{-1} \mathrm{FW}\right)\end{array}$ & $\begin{array}{c}\mathrm{H}_{2} \mathrm{O}_{2} \\
\left(\mu \mathrm{mol} \mathrm{g}{ }^{-1}\right. \\
F W)\end{array}$ \\
\hline Salinity & Foliar spray & \multicolumn{6}{|c|}{ 1st Season } \\
\hline \multirow{5}{*}{ Control } & Distilled water & $2.05 \pm 0.07^{\mathrm{d}}$ & $1.89 \pm 0.01^{\mathrm{d}}$ & $1.23 \pm 0.03^{h}$ & $2.33 \pm 0.06^{\mathrm{d}}$ & $0.460 \pm 0.02^{i}$ & $5.55 \pm 0.1^{\mathrm{i}}$ \\
\hline & Se $(2 \mu \mathrm{M})$ & $2.15 \pm 0.08^{c}$ & $1.94 \pm 0.02^{c}$ & $1.22 \pm 0.02^{h}$ & $2.36 \pm 0.07^{c}$ & $0.440 \pm 0.01^{j}$ & $5.01 \pm 0.2^{j}$ \\
\hline & Se $(4 \mu \mathrm{M})$ & $2.20 \pm 0.06^{b}$ & $2.02 \pm 0.02^{b}$ & $1.20 \pm 0.04^{h}$ & $2.43 \pm 0.08^{b}$ & $0.420 \pm 0.03^{\mathrm{k}}$ & $4.55 \pm 0.09^{k}$ \\
\hline & Se $(8 \mu \mathrm{M})$ & $2.29 \pm 0.09^{a}$ & $2.07 \pm 0.05^{\mathrm{a}}$ & $1.19 \pm 0.02^{h}$ & $2.48 \pm 0.09^{a}$ & $0.403 \pm 0.01^{1}$ & $4.20 \pm 0.08^{1}$ \\
\hline & Distilled water & $1.43 \pm 0.06^{g}$ & $1.68 \pm 0.008^{h}$ & $6.37 \pm 0.02^{e}$ & $1.72 \pm 0.01 \mathrm{~g}$ & $0.583 \pm 0.03^{\mathrm{e}}$ & $10.8 \pm 0.2^{\mathrm{e}}$ \\
\hline \multirow{3}{*}{$\mathrm{NaCl}(200 \mathrm{mM})$} & Se $(2 \mu \mathrm{M})$ & $1.47 \pm 0.05^{\mathrm{fg}}$ & $1.72 \pm 0.008^{g}$ & $6.13 \pm 0.04$ ef & $1.74 \pm 0.03^{\mathrm{f}}$ & $0.566 \pm 0.02^{\mathrm{f}}$ & $8.44 \pm 0.2 \mathrm{~g}$ \\
\hline & Se $(4 \mu \mathrm{M})$ & $1.50 \pm 0.07^{\mathrm{f}}$ & $1.75 \pm 0.009^{\mathrm{f}}$ & $5.75 \pm 0.02^{\mathrm{f}}$ & $1.76 \pm 0.05^{f}$ & $0.540 \pm 0.04 \mathrm{~g}$ & $7.95 \pm 0.3 \mathrm{~g}$ \\
\hline & Se $(8 \mu \mathrm{M})$ & $1.58 \pm 0.04 \mathrm{e}$ & $1.77 \pm 0.008^{\mathrm{e}}$ & $5.17 \pm 0.04 \mathrm{~g}$ & $1.78 \pm 0.02^{\mathrm{e}}$ & $0.510 \pm 0.03^{h}$ & $7.07 \pm 0.2^{h}$ \\
\hline \multirow{5}{*}{$\mathrm{NaCl}(250 \mathrm{mM})$} & Distilled water & $1.10 \pm 0.02^{k}$ & $1.14 \pm 0.009^{1}$ & $9.72 \pm 0.03^{\mathrm{a}}$ & $1.22 \pm 0.01^{\mathrm{k}}$ & $0.713 \pm 0.04^{\mathrm{a}}$ & $16.9 \pm 0.4^{\mathrm{a}}$ \\
\hline & Se $(2 \mu \mathrm{M})$ & $1.17 \pm 0.01^{\mathrm{j}}$ & $1.20 \pm 0.008^{k}$ & $8.21 \pm 0.06^{b}$ & $1.24 \pm 0.03^{j}$ & $0.673 \pm 0.04^{b}$ & $13.4 \pm 0.3^{b}$ \\
\hline & Se $(4 \mu \mathrm{M})$ & $1.24 \pm 0.02^{\mathrm{i}}$ & $1.35 \pm 0.03^{j}$ & $7.71 \pm 0.07^{c}$ & $1.36 \pm 0.02^{\mathrm{i}}$ & $0.643 \pm 0.03^{c}$ & $12.7 \pm 0.2^{c}$ \\
\hline & Se $(8 \mu \mathrm{M})$ & $1.31 \pm 0.03^{\mathrm{h}}$ & $1.40 \pm 0.02^{\mathrm{i}}$ & $7.17 \pm 0.06^{\mathrm{d}}$ & $1.47 \pm 0.03^{\mathrm{h}}$ & $0.610 \pm 0.02^{\mathrm{d}}$ & $11.1 \pm 0.4^{\mathrm{d}}$ \\
\hline & & \multicolumn{6}{|c|}{ 2nd Season } \\
\hline \multirow{5}{*}{ Control } & Distilled water & $2.11 \pm 0.05^{\mathrm{d}}$ & $2.39 \pm 0.02^{\mathrm{d}}$ & $1.21 \pm 0.01^{\mathrm{h}}$ & $1.97 \pm 0.02^{\mathrm{d}}$ & $0.440 \pm 0.01^{\mathrm{h}}$ & $5.43 \pm 0.09^{h}$ \\
\hline & Se $(2 \mu \mathrm{M})$ & $2.21 \pm 0.06^{c}$ & $2.43 \pm 0.02^{c}$ & $1.20 \pm 0.02^{h}$ & $2.03 \pm 0.03^{c}$ & $0.420 \pm 0.02^{i}$ & $4.89 \pm 0.08^{\mathrm{i}}$ \\
\hline & Se $(4 \mu \mathrm{M})$ & $2.26 \pm 0.07^{b}$ & $2.49 \pm 0.009^{b}$ & $1.18 \pm 0.03^{h}$ & $2.10 \pm 0.04^{b}$ & $0.400 \pm 0.01^{j}$ & $4.43 \pm 0.09^{\mathrm{j}}$ \\
\hline & Se $(8 \mu \mathrm{M})$ & $2.35 \pm 0.07^{\mathrm{a}}$ & $2.54 \pm 0.007^{\mathrm{a}}$ & $1.17 \pm 0.01^{\mathrm{h}}$ & $2.16 \pm 0.06^{\mathrm{a}}$ & $0.383 \pm 0.01^{\mathrm{k}}$ & $4.08 \pm 0.08^{k}$ \\
\hline & Distilled water & $1.46 \pm 0.02^{g}$ & $1.76 \pm 0.009 \mathrm{~g}$ & $5.96 \pm 0.02^{\mathrm{e}}$ & $0.29 \pm 0.009 \mathrm{~g}$ & $0.543 \pm 0.02^{\mathrm{d}}$ & $10.5 \pm 0.2^{\mathrm{d}}$ \\
\hline \multirow{3}{*}{$\mathrm{NaCl}(200 \mathrm{mM})$} & Se $(2 \mu \mathrm{M})$ & $1.51 \pm 0.03^{f}$ & $1.78 \pm 0.008^{f}$ & $5.72 \pm 0.03^{\text {ef }}$ & $0.313 \pm 0.008^{g}$ & $0.526 \pm 0.03^{\mathrm{e}}$ & $8.12 \pm 0.1^{e}$ \\
\hline & Se $(4 \mu \mathrm{M})$ & $1.54 \pm 0.03^{f}$ & $1.80 \pm 0.009^{f}$ & $5.34 \pm 0.03^{f}$ & $0.336 \pm 0.009^{f}$ & $0.500 \pm 0.02^{f}$ & $7.63 \pm 0.2^{f}$ \\
\hline & Se $(8 \mu \mathrm{M})$ & $1.62 \pm 0.02^{\mathrm{e}}$ & $1.82 \pm 0.01^{\mathrm{e}}$ & $4.76 \pm 0.04 \mathrm{~g}$ & $0.383 \pm 0.04^{\mathrm{e}}$ & $0.470 \pm 0.02 \mathrm{~g}$ & $6.75 \pm 0.09 \mathrm{~g}$ \\
\hline \multirow{4}{*}{$\mathrm{NaCl}(250 \mathrm{mM})$} & Distilled water & $1.12 \pm 0.01^{\mathrm{k}}$ & $1.24 \pm 0.008^{k}$ & $9.24 \pm 0.05^{\mathrm{a}}$ & $0.136 \pm 0.01^{\mathrm{k}}$ & $0.663 \pm 0.03^{a}$ & $16.3 \pm 0.2^{\mathrm{a}}$ \\
\hline & Se $(2 \mu \mathrm{M})$ & $1.19 \pm 0.03^{j}$ & $1.26 \pm 0.01^{j}$ & $7.69 \pm 0.07^{b}$ & $0.1667 \pm 0.01^{j}$ & $0.623 \pm 0.04^{\mathrm{b}}$ & $12.9 \pm 0.2^{b}$ \\
\hline & Se $(4 \mu \mathrm{M})$ & $1.26 \pm 0.02^{\mathrm{i}}$ & $1.38 \pm 0.03^{\mathrm{i}}$ & $7.19 \pm 0.05^{c}$ & $0.190 \pm 0.02^{\mathrm{i}}$ & $0.593 \pm 0.02^{c}$ & $12.2 \pm 0.1^{c}$ \\
\hline & Se $(8 \mu \mathrm{M})$ & $1.33 \pm 0.01^{\mathrm{h}}$ & $1.49 \pm 0.02^{h}$ & $6.65 \pm 0.07^{d}$ & $0.223 \pm 0.03^{h}$ & $0.560 \pm 0.01^{\mathrm{d}}$ & $10.6 \pm 0.2^{\mathrm{d}}$ \\
\hline
\end{tabular}

Means followed by the same letters are not significantly different by LSD $(p<0.05)$.

\subsection{Interrelationship among Studied Parameters}

The interrelationship among evaluated parameters was investigated using principal component analysis. The first two principal components explained about $92.48 \%$ of the variability $(80.51 \%$ by PC1 and $11.97 \%$ by PC2) (Figure 3$)$. The studied parameters represented by parallel vectors indicated a strong positive relationship, while those placed nearly opposite exhibited a highly negative association. The investigated parameters could be divided into two groups: the first group contained all agronomic and growth traits, photosynthetic pigments, gas exchange and plant water relations, while the second group was comprised of enzymatic and non-enzymatic antioxidants, sodium, superoxide radical and hydrogen peroxide. A strong positive association was determined among the traits in each group, while a negative association was detected between the first and second group. Furthermore, PC1 separated salinity treatments into two groups: non-stressed treatments are located on the positive side, while those under a high salinity level are located on the negative side. The traits in the second group are associated with the high salt stress treatment. Likewise, PC2 separated Se foliar applications. Foliar spray with distilled water (DW) displayed the highest negative scores on PC2, while Se foliar application using $8 \mu \mathrm{M}$ exhibited the highest scores. 


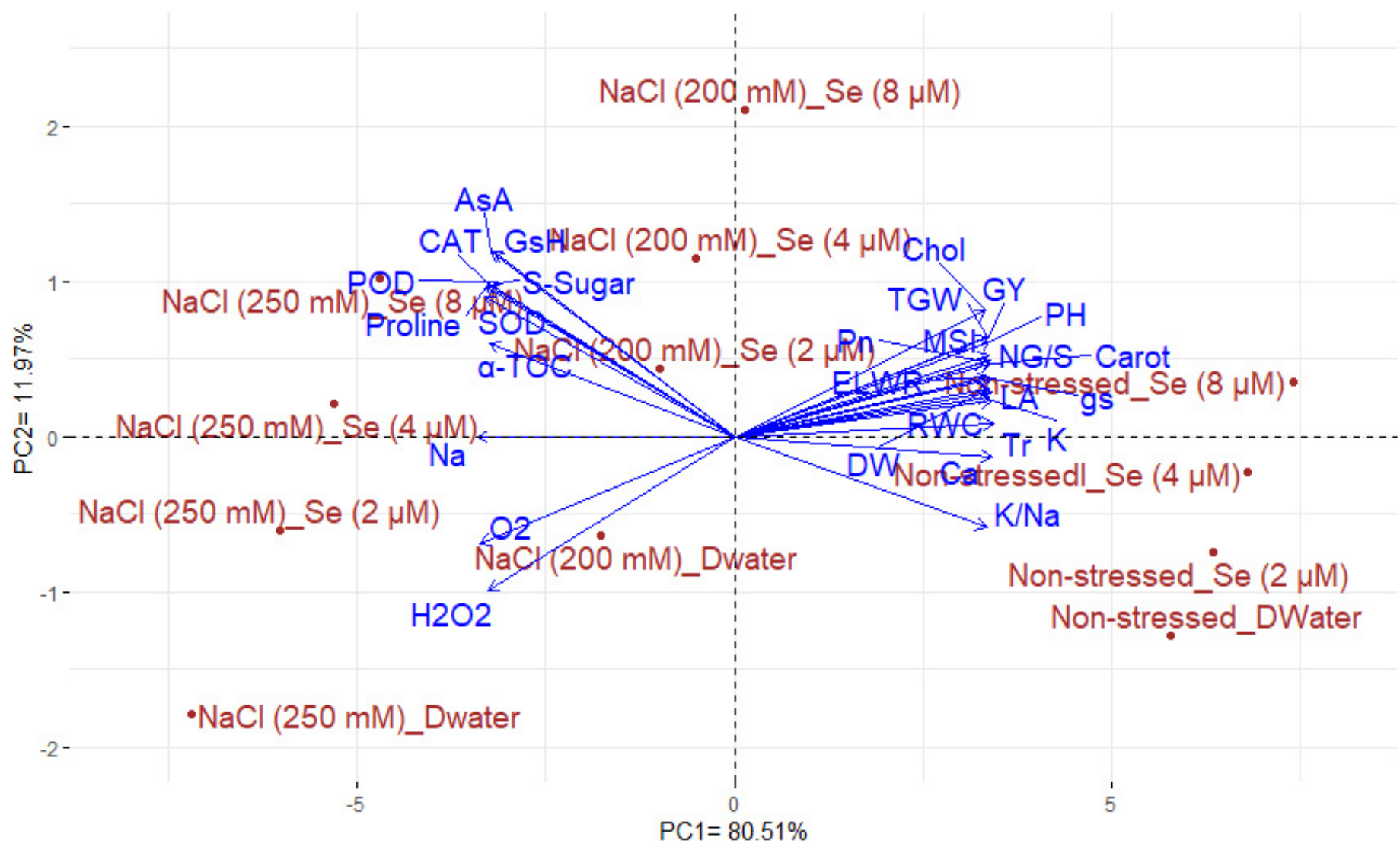

Figure 3. Biplot of principal component analysis displaying the relationship among the evaluated parameters. Chol: total chlorophylls, Carot: total carotenoids, Pn: net photosynthetic rate, Tr: transpiration rate, gs: stomatal conductance, RWC: relative water content, MSI: membrane stability index, ELWR: excised leaf water retention, Proline: proline content, S-Sugar: total soluble sugars, CAT: catalase, POD: peroxidase, SOD: superoxide dismutase, AsA: ascorbate, GSH: total glutathione, $\alpha$-TOC: $\alpha$-tocopherol, Ca: calcium, K: potassium, Na: sodium, O2: superoxide radical, $\mathrm{H} 2 \mathrm{O} 2$ : hydrogen peroxide, $\mathrm{PH}$ : plant height, DW: shoot dry weight, LA: leaf area, MG/S: number of grains/spike, TGW: thousand-grain weight, GY: grain yield/plant.

\section{Discussion}

The present study demonstrated that soil salinity (200 and $250 \mathrm{mM} \mathrm{NaCl}$ ) caused a considerable reduction in plant productivity (Figures 1 and 2). This reduction is attributed to the influence of osmotic pressure produced by salinity worsening metabolic functions and to the reduction in energy requirements and cellular divisions [48-50]. Salinity adverse effects led to a reduction in leaf photosynthetic pigments, photosynthetic efficiency, stomatal closure, imbalance in ionic and gas exchange, toxic ion uptake and, subsequently, growth inhibition [3,51]. Damaging chlorophyll levels and plasma membranes result from oxidative stress by excess ROS [52]. Oxidative stress is related to diminished Mg accumulation and increased $\mathrm{Na}^{+}$uptake, which affect the synthesis of the chlorophyll molecule. The exposure of wheat to salinity stress leads to alterations in the pigment-protein complex [53]. The accumulation of salt decreases the synthesis of pigments, which leads to a reduction in enzyme activity [54]. In addition to increasing the activity of chlorophyllase [55], this decreases leaf water potential, $\mathrm{N}$-uptake and photosynthetic efficiency $[56,57]$. On the other hand, plants have advanced antioxidant systems to attenuate the destructive impacts of ROS [58]. This system includes many enzymatic and non-enzymatic compounds, such as proline, ascorbic acid and carotenoids [59,60].

In the present study, wheat plants treated by Se as an exogenous growth promotion presented better performance under salinity stress compared with untreated ones. Se foliar 
application enhanced wheat growth and yield under salinity by ameliorating water status; postponing senescence in plants [61,62]; improving cell size or cell number; protecting cell turgidity, which can lead to cell elongation [63]; boosting mature leaf rigidity [64,65]; improving photosynthetic attributes, such as internal $\mathrm{CO}_{2}$ concentration and stomatal conductance [66]; and, subsequently, enhancing photosynthetic efficiency [67]. Accordingly, Se foliar application could be considered an efficient bio-stimulant that improves wheat salt tolerance under salinity stress conditions [68]. Figure 4 is a schematic diagram explaining the mechanisms of Se to alleviate the devastating impacts of salt stress on wheat plants.
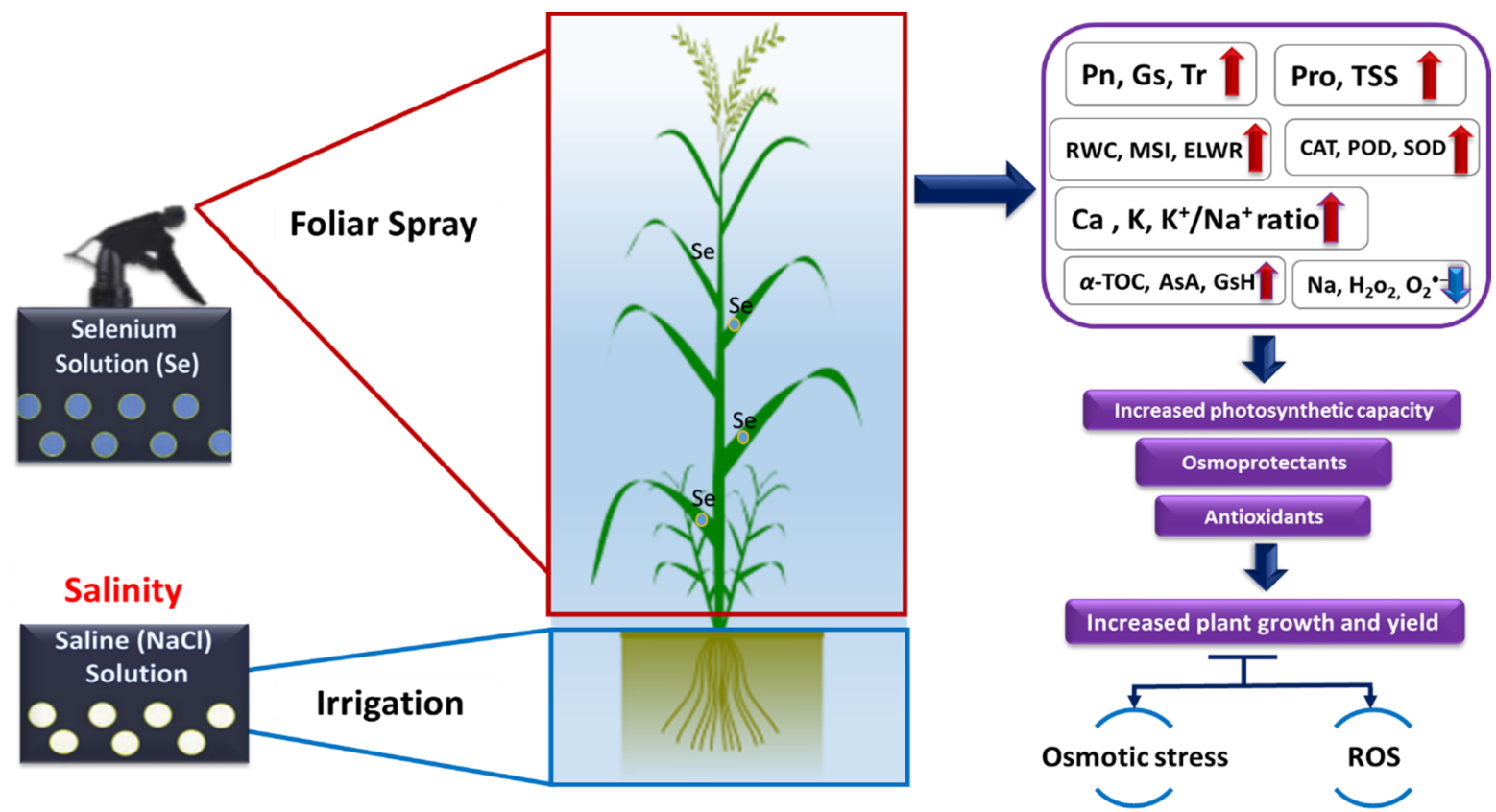

Figure 4. Schematic diagram explaining the mechanisms of Se in alleviating the devastating impacts of salt stress on wheat plants. Application of Se could enhance plant growth and yield attributes by (i) improving photosynthetic pigments and increasing photosynthetic efficiency; (ii) elevating proline and total soluble sugar contents for superior osmoprotection; (iii) enhancing antioxidant system to protect the membrane stability index (MSI), stomatal conductance (gs), relative water content (RWC), excised leaf water retention (ELWR), potassium (K), calcium (Ca), net photosynthetic rate (Pn), glutathione (GSH), ascorbic acid (AsA), $\alpha$-tocopherol ( $\alpha$-TOC), proline (Pro), total soluble sugar (TSS), catalase (CAT), superoxide dismutase (SOD), peroxidase (POX), sodium (Na) and hydrogen peroxide $\left(\mathrm{H}_{2} \mathrm{O}_{2}\right)$.

There are various researchers who used Se foliar spray to improve plant growth under diverse stresses in different crops, e.g., sorghum under heat stress [69], tomato under salinity stress [24], wheat under drought stress [70], wheat under heat stress [62] and maize under salinity stress [63]. Alyemeni et al. [71] depicted that the quick elimination of ROS and nutrients accumulation was due to the Se amelioration of photosynthetic inhibition. Additionally, Elkelish et al. [55] documented that exogenous Se application enhanced chlorophyll and carotenoid pigments and had a positive effect on stomatal conductance and photosynthetic efficiency. Moreover, Iqbal et al. [62] demonstrated that the Se application with an optimal amount led to increased chlorophyll and protected the chloroplast structure against oxidative destruction. Decreasing oxidative damage by ROS resulted in a positive impact of Se application on chlorophyll content and its role in the chloroplast structure $[27,63]$. Moreover, Se application increased chlorophyll content in various crops, such as sorghum [69], maize [72] and spinach [73]. Indeed, low concentrations were used to stimulate plant growth and yield because high doses have toxic effects [63,65]. Hawrylak-Nowak [74] found that applying high concentrations of Se in maize led to decreased growth and biomass resulting from the accumulation of high phosphorus in the straw of plants. 
The results of the current study suggest that Se application increased stomatal conductance (gs), photosynthetic effectiveness, net photosynthesis (Pn) and transpiration (Tr) rates and salt-stressed leaf gas exchange (Table 1). The supplementation of Se in the optimal rate increased photosynthetic functioning by improving chlorophyll fluorescence characteristics, $\mathrm{CO}_{2}$ assimilation and the photosynthetic rate under salinity stress [71]. In addition, Se application significantly increased water relations (i.e., RWC, MSI and ELWR) in comparison with untreated plants, and the highest values were attributed to the high dose of $8 \mu \mathrm{M}$ (Table 2).

Salinity stress disturbed water status and decreased the hydraulic conductivity of the root, which resulted in a diminished level of water uptake from roots to shoots [75-78]. Furthermore, salinity reduced RWC and led to a decrease in water uptake with toxic effects on stressed plants. On the other hand, the results show that Se application improved RWC via the accumulation of sugars reflecting the level of plant productivity under stress conditions [79]. Se increased proline accumulation, which subsequently improved photosynthetic efficiency, ATP production and water use efficiency, as reported previously by Guo et al. [80]. Proline is a non-enzymatic antioxidants and has a vital role in osmotic adjustment under salinity stress [55,81-84]. It diminishes the negative impacts of ROS and boosts plant tolerance by diminishing the detoxification of ROS produced due to salt stress [85]. Moreover, proline improves plant antioxidant systems [86,87]. Chandrashekar and Sandhyarani [88] reported that proline accumulation assisted plants to recompense energy and increased their survival under salinity stress. Furthermore, Chun et al. [89] manifested that proline content is considered an optimistic indicator for recognizing salinity stress, particularly under high levels. The current study proved that the proline concentration in wheat leaves under salinity stress increased considerably when compared with non-stressed plants. The maximum concentration of proline was detected in plants treated with $8 \mu \mathrm{M}$ Se (Table 2). Se regulated the accumulation of proline and led to elevated activity of the $\gamma$-GK enzyme and decreased PROX activity [90]. Similarly, it prevented photosynthetic arrest by producing RWC and protected Rubisco, which produced photosynthetic apparatus, maintained proline and soluble sugar contents, and also scavenged ROS [78,91]. Similar findings were obtained in wheat plants treated with Se under cadmium stress by Khan et al. [90]. Likewise, Hawrylak-Nowak [28] documented that Se contributed to an increasing proline concentration compared with untreated plants under salinity conditions. Consequently, Se can adjust the accumulation of free proline in plants exposed to salinity stress. Similarly, the application of Se leads to an increase in the concentration of soluble sugars (Table 2). Elkelish et al. [55],Ahanger and Agarwal [78] elucidated that soluble sugar accumulation has a great role in providing ionic balance and osmotic in plant cells and, subsequently, leads to stress tolerance.

The ROS caused by salinity stress can be constrained by antioxidant enzymatic defense systems (SOD, CAT and POX) or non-enzymatic defense systems (GSH, AsA and $\alpha$ tocopherol) $[92,93]$. SOD could be the first line of protection against ROS $[55,94]$. It inhibits the formation of the hydroxyl $\left(\mathrm{OH}^{-}\right)$radical that leads to the high production of chloroplast function [71]. Moreover, CAT has a vital role in eliminating $\mathrm{H}_{2} \mathrm{O}_{2}$ as it scavenges ROS in leaves. Likewise, it obstructs the formation of hydroxyl radicals that lead to a decline in the lipid peroxidation of cell membranes [94]. Moreover, POX has a major role in eliminating $\mathrm{H}_{2} \mathrm{O}_{2}$ and diminishing oxidative damage in plant tissues $[95,96]$. Moreover, AsA is an effective ROS scavenger due to its competence in providing enzymatic and nonenzymatic responses to control the level of $\mathrm{H}_{2} \mathrm{O}_{2}$ and protect cell membranes by scavenging $\mathrm{O}_{2}{ }^{\bullet-}$ and $\mathrm{OH}^{-}$[60,97-99]. Similarly, $\alpha$-TOC content has an important role in antioxidant systems owing to its function in oxidative stress [93]. The obtained findings suggest that Se application ameliorated the activities of CAT, POX and SOD that, in turn, upregulated the AsA-GSH pathway. Subsequently, this led to the protection of the photosynthesis process by inhibiting toxic radicals and maintaining the NADP level. Additionally, Se application led to a reduction in ROS levels and enhanced growth by inducing enzymatic and non-enzymatic antioxidants and modulated osmolytes levels. Similarly, previous 
studies documented that the application of Se reinforced antioxidant activities in different crops, such as sorrel [27], maize [72] and tomato [71].

Se application prevented $\mathrm{Na}^{+}$accumulation in wheat plants (Table 4). It could be speculated that Se application stimulates the expression of the $\mathrm{Na}^{+} / \mathrm{H}^{+}$antiport that decreases its toxic effects [100]. Furthermore, Se application led to increased uptake of Ca and K. These elements have considerable functions in promoting growth regulation by inference cellular stress signaling, antioxidant metabolism and nitrogen assimilation [78,101,102]. Furthermore, increased uptake of $\mathrm{Ca}$ and $\mathrm{K}$ and a reduction in $\mathrm{Na}^{+}$in wheat led to improved stress signaling and, consequently, increased salt tolerance [55]. Astaneh et al. [26] found that Se foliar spray at rates of 4,8 and $16 \mathrm{mg} \mathrm{L}^{-1}$ led to an improvement in the uptake of $\mathrm{K}^{+}$and reduced $\mathrm{Na}+$ under salinity stress. Se has a positive impact on decreasing the uptake of $\mathrm{Na}^{+}$and increasing $\mathrm{K}^{+}$uptake at the membrane transport level [101]. Hence, the $\mathrm{K}^{+} / \mathrm{Na}^{+}$ratio increased, which led to the protection of vital processes and maintenance of the osmotic balance [102].

Salinity stress reduces membrane stability due to $\mathrm{H}_{2} \mathrm{O}_{2}$-mediated membrane peroxidation [103], polyunsaturated fatty acids [104] and lipoxygenase activity [105]. Furthermore, the transfer of the electrons $\mathrm{O}_{2}{ }^{--}, \mathrm{H}_{2} \mathrm{O}_{2}$ and $\mathrm{OH}^{\bullet-}$ has a negative effect on proteins, DNA and lipids [7]. Consequently, the safety of cell membranes and the photosynthesis process are affected [106]. In the present study, the contents of reactive species $\mathrm{O}_{2}{ }^{\bullet-}$ and $\mathrm{H}_{2} \mathrm{O}_{2}$ increased under soil salinity. However, Se application reduced their content in treated plants (Table 4). Se application limited the overexpression of lipoxygenase to maintain the fatty acid composition and also decreased ROS production, which regulates the antioxidant systems to decrease $\mathrm{H}_{2} \mathrm{O}_{2}$ generation $[107,108]$.

\section{Conclusions}

The obtained results indicate that salt stress mainly reduced the photosynthetic pigments, photosynthetic efficiency and mineral uptake and accordingly reduced wheat growth and grain yield. On the contrary, Se application (in particular $8 \mu \mathrm{M}$ ) ameliorated photosynthetic efficiency, antioxidants defense system and osmolytes metabolism, and improved growth and yield productivity. Consequently, Se application as foliar spray displayed a valuable role in mitigating the devastating impacts of salinity stress on physiobiochemical and growth attributes and grain yield of bread wheat. Therefore, Se application (in particular $8 \mu \mathrm{M}$ ) could be recommended as a significant approach for enhancing wheat growth and productivity under salinity stress.

Author Contributions: Conceptualization, E.-S.M.D., A.-R.M.A.M., M.F.A.E.-M., E.M., S.M.A.I.A., M.F.A., M.F.R. and S.A.I.; methodology, E.-S.M.D., A.-R.M.A.M., M.F.A.E.-M., E.M., S.M.A.I.A. and S.A.I.; software, E.-S.M.D., A.-R.M.A.M., M.F.A.E.-M. and E.M.; validation, E.-S.M.D., A.-R.M.A.M., M.F.A.E.-M., E.M., S.M.A.I.A. and S.A.I.; formal analysis, E.-S.M.D., A.-R.M.A.M., M.F.A.E.-M., E.M., S.M.A.I.A. and S.A.I.; investigation, E.-S.M.D., A.-R.M.A.M., M.F.A.E.-M., E.M., S.M.A.I.A. and S.A.I.; resources, E.-S.M.D., M.F.A.E.-M., M.F.A. and M.F.R.; data curation, E.-S.M.D., A.-R.M.A.M., M.F.A.E.-M., E.M., S.M.A.I.A., M.F.A., S.A.I. and M.F.R.; writing—original draft preparation, E.-S.M.D., A.-R.M.A.M., M.F.A.E.-M., E.M., S.M.A.I.A. and S.A.I.; writing-review and editing, E.-S.M.D., A.-R.M.A.M., M.F.A.E.-M., E.M., S.M.A.I.A., M.F.A., M.F.R. and S.A.I.; visualization, E.-S.M.D., A.-R.M.A.M., M.F.A.E.-M., E.M., S.M.A.I.A., M.F.A., M.F.R. and S.A.I.; supervision, E.-S.M.D., A.-R.M.A.M., M.F.A.E.-M., E.M., S.M.A.I.A., M.F.A., M.F.R. and S.A.I. All authors have read and agreed to the published version of the manuscript.

Funding: This research received no external funding.

Institutional Review Board Statement: Not applicable.

Informed Consent Statement: Not applicable.

Data Availability Statement: The data presented in this study are available upon request from the corresponding author. 
Acknowledgments: The authors thank the Taif University for funding this work through Taif University Researchers Supporting Project number (TURSP -2020/111), Taif University, Taif, Saudi Arabia.

Conflicts of Interest: The authors declare no conflict of interest.

\section{References}

1. FAOSTAT. Food and Agriculture Organization of the United Nations. Statistical Database. Available online: http://www.fao.org/ faostat/en/\#data (accessed on 13 February 2021).

2. Moustafa, E.S.; El-Sobky, E.-S.E.; Farag, H.I.; Yasin, M.A.; Attia, A.; Rady, M.O.; Awad, M.F.; Mansour, E. Sowing date and genotype influence on yield and quality of dual-purpose barley in a salt-affected arid region. Agronomy 2021, 11, 717. [CrossRef]

3. Mansour, E.; Moustafa, E.S.; Desoky, E.-S.M.; Ali, M.; Yasin, M.A.; Attia, A.; Alsuhaibani, N.; Tahir, M.U.; El-Hendawy, S. Multidimensional evaluation for detecting salt tolerance of bread wheat genotypes under actual saline field growing conditions. Plants 2020, 9, 1324. [CrossRef] [PubMed]

4. Awaad, H.A.; Mansour, E.; Akrami, M.; Fath, H.E.; Javadi, A.A.; Negm, A. Availability and Feasibility of Water Desalination as a Non-Conventional Resource for Agricultural Irrigation in the MENA Region: A Review. Sustainability 2020, 12, 7592. [CrossRef]

5. Abd El-Mageed, T.A.; Rady, M.O.; Semida, W.M.; Shaaban, A.; Mekdad, A.A. Exogenous micronutrients modulate morphophysiological attributes, yield, and sugar quality in two salt-stressed sugar beet cultivars. J. Plant Nutr. Soil Sci. 2021, 1-16. [CrossRef]

6. Munns, R. Comparative physiology of salt and water stress. Plant Cell Environ. 2002, 25, 239-250. [CrossRef] [PubMed]

7. Mittler, R. Oxidative stress, antioxidants and stress tolerance. Trends Plant Sci. 2002, 7, 405-410. [CrossRef]

8. Del Buono, D.; Regni, L.; Del Pino, A.M.; Bartucca, M.L.; Palmerini, C.A.; Proietti, P. Effects of megafol on the olive cultivar 'Arbequina' grown under severe saline stress in terms of physiological traits, oxidative stress, antioxidant defenses, and cytosolic $\mathrm{Ca}^{2+}$. Front. Plant Sci. 2020, 11, 603576. [CrossRef]

9. Singh, P.; Mahajan, M.M.; Singh, N.K.; Kumar, D.; Kumar, K. Physiological and molecular response under salinity stress in bread wheat (Triticum aestivum L.). J. Plant Biochem. Biot. 2020, 29, 125-133. [CrossRef]

10. Marschner, H. Functions of Mineral Nutrients. In Mineral Nutrition of Higher Plants; Elsevier: Amsterdam, The Netherlands, 1995; pp. 229-312.

11. Rady, M.M. Effect of 24-epibrassinolide on growth, yield, antioxidant system and cadmium content of bean (Phaseolus vulgaris L.) plants under salinity and cadmium stress. Sci. Hortic. 2011, 129, 232-237. [CrossRef]

12. Sudhir, P.; Murthy, S.D.S. Effects of salt stress on basic processes of photosynthesis. Photosynthetica 2004, 42, 481-486. [CrossRef]

13. Bethke, P.C.; Drew, M.C. Stomatal and nonstomatal components to inhibition of photosynthesis in leaves of Capsicum annuum during progressive exposure to $\mathrm{NaCl}$ Salinity. Plant Physiol. 1992, 99, 219-226. [CrossRef] [PubMed]

14. Regni, L.; Del Pino, A.M.; Mousavi, S.; Palmerini, C.A.; Baldoni, L.; Mariotti, R.; Mairech, H.; Gardi, T.; D'Amato, R.; Proietti, P. Behavior of four olive cultivars during salt stress. Front. Plant Sci. 2019, 10, 867. [CrossRef]

15. Kahrizi, S.; Sedighi, M.; Sofalian, O. Effect of salt stress on proline and activity of antioxidant enzymes in ten durum wheat cultivars. Ann. Biol. Res. 2012, 3, 3870-3874

16. Acosta-Motos, J.R.; Ortuño, M.F.; Bernal-Vicente, A.; Diaz-Vivancos, P.; Sanchez-Blanco, M.J.; Hernandez, J.A. Plant responses to salt stress: Adaptive mechanisms. Agronomy 2017, 7, 18. [CrossRef]

17. El-Sanatawy, A.M.; El-Kholy, A.S.; Ali, M.; Awad, M.F.; Mansour, E. Maize seedling establishment, grain yield and crop water productivity response to seed priming and irrigation management in a mediterranean arid environment. Agronomy 2021, 11, 756. [CrossRef]

18. Mansour, E.; Moustafa, E.S.; El-Naggar, N.Z.; Abdelsalam, A.; Igartua, E. Grain yield stability of high-yielding barley genotypes under Egyptian conditions for enhancing resilience to climate change. Crop Pasture Sci. 2018, 69, 681-690. [CrossRef]

19. Shardendu; Salhani, N.; Boulyga, S.F.; Stengel, E. Phytoremediation of selenium by two helophyte species in subsurface flow constructed wetland. Chemosphere 2003, 50, 967-973. [CrossRef]

20. Pennanen, A.; Xue, T.; Hartikainen, H. Protective role of selenium in plant subjected to severe UV irradiation stress. J. Appl. Bot. 2002, 76, 66-76.

21. Rady, M.O.; Semida, W.M.; Abd El-Mageed, T.A.; Howladar, S.M.; Shaaban, A. Foliage applied selenium improves photosynthetic efficiency, antioxidant potential and wheat productivity under drought stress. Int. J. Agric. Biol. 2020, 24, 1293-1300.

22. Hasanuzzaman, M.; Hossain, M.A.; Fujita, M. Selenium-induced up-regulation of the antioxidant defense and methylglyoxal detoxification system reduces salinity-induced damage in rapeseed seedlings. Biol. Trace Elem. 2011, 143, 1704-1721. [CrossRef]

23. D'Amato, R.; De Feudis, M.; Hasuoka, P.E.; Regni, L.; Pacheco, P.H.; Onofri, A.; Businelli, D.; Proietti, P. The selenium supplementation influences olive tree production and oil stability against oxidation and can alleviate the water deficiency effects. Front. Plant Sci. 2018, 9, 1191. [CrossRef] [PubMed]

24. Diao, M.; Ma, L.; Wang, J.; Cui, J.; Fu, A.; Liu, H.-Y. Selenium promotes the growth and photosynthesis of tomato seedlings under salt stress by enhancing chloroplast antioxidant defense system. J. Plant Growth Regul. 2014, 33, 671-682. [CrossRef]

25. Regni, L.; Palmerini, C.A.; Del Pino, A.M.; Businelli, D.; D’Amato, R.; Mairech, H.; Marmottini, F.; Micheli, M.; Pacheco, P.H.; Proietti, P. Effects of selenium supplementation on olive under salt stress conditions. Sci. Hortic. 2021, 278, 109866. [CrossRef]

26. Astaneh, R.K.; Bolandnazar, S.; Nahandi, F.Z.; Oustan, S. The effects of selenium on some physiological traits and K, Na concentration of garlic (Allium sativum L.) under $\mathrm{NaCl}$ stress. Inf. Process. Agric. 2018, 5, 156-161. [CrossRef] 
27. Kong, L.; Wang, M.; Bi, D. Selenium modulates the activities of antioxidant enzymes, osmotic homeostasis and promotes the growth of sorrel seedlings under salt stress. Plant Growth Regul. 2005, 45, 155-163. [CrossRef]

28. Hawrylak-Nowak, B. Beneficial effects of exogenous selenium in cucumber seedlings subjected to salt stress. Biol. Trace Elem. Res. 2009, 132, 259-269. [CrossRef]

29. Hoagland, D.R.; Arnon, D.I. The water-culture method for growing plants without soil. Circ. Calif. Agric. Exp. Stn. 1950, 347, 1-39.

30. Fadeel, A.A. Location and properties of chloroplasts and pigment determination in roots. Physiol. Plant. 1962, 15, 130-146. [CrossRef]

31. Barrs, H.D.; Weatherley, P.E. A re-examination of the relative turgidity technique for estimating water deficits in leaves. Aust. J. Biol. Sci. 1962, 15, 413-428. [CrossRef]

32. Premachandra, G.S.; Saneoka, H.; Ogata, S. Cell membrane stability, an indicator of drought tolerance, as affected by applied nitrogen in soyabean. J. Agric. Sci. 1990, 115, 63-66. [CrossRef]

33. Farshadfar, E.; Afarinesh, A.; Sutka, J. Inheritance of drought tolerance in Maize. Cereal Res. Commun. 2002, 30, $285-291$. [CrossRef]

34. Irigoyen, J.J.; Emerich, D.W.; Sanchez-Diaz, M. Water stress induced changes in concentrations of proline and total soluble sugars in nodulated alfalfa (Medicago sativa) plants. Physiol. Plant. 1992, 84, 55-60. [CrossRef]

35. Bates, L.S.; Waldren, R.P.; Teare, I.D. Rapid determination of free proline for water-stress studies. Plant Soil 1973, 39, $205-207$. [CrossRef]

36. Mukherjee, S.P.; Choudhuri, M.A. Implications of water stress-induced changes in the levels of endogenous ascorbic acid and hydrogen peroxide in Vigna seedlings. Physiol. Plant. 1983, 58, 166-170. [CrossRef]

37. Giannopolitis, C.N.; Ries, S.K. Superoxide Dismutases. Plant Physiol. 1977, 59, 309-314. [CrossRef]

38. Aebi, H. Catalase in vitro. In Methods in Enzymology; Elsevier: Amsterdam, The Netherlands, 1984; pp. $121-126$.

39. Maechlay, A.; Chance, B. The assay of catalase and peroxidase. In Methods of Biochemical Analysis; Interscience Inc.: New York, NY, USA, 1954; pp. 357-424.

40. Klapheck, S.; Zimmer, I.; Cosse, H. Scavenging of hydrogen peroxide in the endosperm of Ricinus communis by ascorbate peroxidase. Plant Cell Physiol. 1990, 31, 1005-1013.

41. Kampfenkel, K.; Vanmontagu, M.; Inze, D. Extraction and determination of ascorbate and dehydroascorbate from plant tissue. Anal. Biochem. 1995, 225, 165-167. [CrossRef]

42. Griffith, O.W. Determination of glutathione and glutathione disulfide using glutathione reductase and 2-vinylpyridine. Anal. Biochem. 1980, 106, 207-212. [CrossRef]

43. Konings, E.J.M.; Roomans, H.H.S.; Beljaars, P.R. Liquid chromatographic determination of tocopherols and tocotrienols in margarine, infant foods, and vegetables. J. AOAC Int. 1996, 79, 902-906. [CrossRef]

44. Ching, L.S.; Mohamed, S. Alpha-tocopherol content in 62 edible tropical plants. J. Agric. Food Chem. 2001, 49, 3101-3105. [CrossRef]

45. Velikova, V.; Yordanov, I.; Edreva, A. Oxidative stress and some antioxidant systems in acid rain-treated bean plants. Plant Sci. 2000, 151, 59-66. [CrossRef]

46. Kubiś, J. Exogenous spermidine differentially alters activities of some scavenging system enzymes, $\mathrm{H} 2 \mathrm{O} 2$ and superoxide radical levels in water-stressed cucumber leaves. J. Plant Physiol. 2008, 165, 397-406. [CrossRef]

47. Williams, V.; Twine, S. Flame photometric method for sodium, potassium and calcium. In Modern Methods of Plant Analysis; Peach, K., Tracey, M.V., Eds.; Springer: Berlin, Germany, 1960; pp. 3-5.

48. Zaki, S.S.; Rady, M.M. Moringa oleifera leaf extract improves growth, physiochemical attributes, antioxidant defence system and yields of salt-stressed Phaseolus vulgaris L. plants. Int. J. Chem. Tech. Res. 2015, 8, 120-134.

49. Desoky, E.-S.M.; Elrys, A.S.; Rady, M.M. Integrative moringa and licorice extracts application improves Capsicum annuum fruit yield and declines its contaminant contents on a heavy metals-contaminated saline soil. Ecotoxicol. Environ. Saf. 2019, 169, 50-60. [CrossRef] [PubMed]

50. Moustafa, E.S.; Ali, M.; Kamara, M.M.; Awad, M.F.; Hassanin, A.A.; Mansour, E. Field screening of wheat advanced lines for salinity tolerance. Agronomy 2021, 11, 281. [CrossRef]

51. Rady, M.M.; Desoky, E.S.M.; Elrys, A.S.; Boghdady, M.S. Can licorice root extract be used as an effective natural biostimulant for salt-stressed common bean plants? S. Afr. J. Bot. 2019, 121, 294-305. [CrossRef]

52. Rady, M.M.; Elrys, A.S.; Abo El-Maati, M.F.; Desoky, E.-S.M. Interplaying roles of silicon and proline effectively improve salt and cadmium stress tolerance in Phaseolus vulgaris plant. Plant Physiol. Biochem. 2019, 139, 558-568. [CrossRef]

53. Levitt, J. Responses of Plants to Environmental Stress, vol.2: Water, Radiation, Salt, and Other Stresses; Academic Press: New York, NY, USA, 1980.

54. Pattanayak, G.K.; Tripathy, B.C. Overexpression of protochlorophyllide oxidoreductase C regulates oxidative stress in Arabidopsis. PLoS ONE 2011, 6, e26532. [CrossRef]

55. Elkelish, A.A.; Soliman, M.H.; Alhaithloul, H.A.; El-Esawi, M.A. Selenium protects wheat seedlings against salt stress-mediated oxidative damage by up-regulating antioxidants and osmolytes metabolism. Plant Physiol. Biochem. 2019, 137, 144-153. [CrossRef] [PubMed]

56. Ahanger, M.A.; Tomar, N.S.; Tittal, M.; Argal, S.; Agarwal, R.M. Plant growth under water/salt stress: ROS production; antioxidants and significance of added potassium under such conditions. Physiol. Mol. Biol. Plants 2017, 23, 731-744. [CrossRef] 
57. Khan, M.I.R.; Asgher, M.; Khan, N.A. Alleviation of salt-induced photosynthesis and growth inhibition by salicylic acid involves glycinebetaine and ethylene in mungbean (Vigna radiata L.). Plant Physiol. Biochem. 2014, 80, 67-74. [CrossRef]

58. Halliwell, B.; Gutteridge, J.M.C. Free Radicals in Biology and Medicine; Oxford University Press: Oxford, UK, 2015. [CrossRef]

59. Schutzendubel, A. Plant responses to abiotic stresses: Heavy metal-induced oxidative stress and protection by mycorrhization. J. Exp. Bot. 2002, 53, 1351-1365. [CrossRef] [PubMed]

60. Semida, W.M.; Rady, M.M. Presoaking application of propolis and maize grain extracts alleviates salinity stress in common bean (Phaseolus vulgaris L.). Sci. Hortic. 2014, 168, 210-217. [CrossRef]

61. Djanaguiraman, M.; Devi, D.D.; Shanker, A.K.; Sheeba, J.A.; Bangarusamy, U. Selenium-An antioxidative protectant in soybean during senescence. Plant Soil 2005, 272,77-86. [CrossRef]

62. Iqbal, M.; Hussain, I.; Liaqat, H.; Ashraf, M.A.; Rasheed, R.; Rehman, A.U. Exogenously applied selenium reduces oxidative stress and induces heat tolerance in spring wheat. Plant Physiol. Biochem. 2015, 94, 95-103. [CrossRef] [PubMed]

63. Jiang, C.; Zu, C.; Lu, D.; Zheng, Q.; Shen, J.; Wang, H.; Li, D. Effect of exogenous selenium supply on photosynthesis, Na ${ }^{+}$ accumulation and antioxidative capacity of maize (Zea mays L.) under salinity stress. Sci. Rep. 2017, 7, 42039. [CrossRef]

64. Kahakachchi, C.; Boakye, H.; Uden, P.; Tyson, J. Chromatographic speciation of anionic and neutral selenium compounds in Se-accumulating Brassica juncea (Indian mustard) and in selenized yeast. J. Chromatogr. A 2004, 1054, 303-312. [CrossRef]

65. Jia, H.; Song, Z.; Wu, F.; Ma, M.; Li, Y.; Han, D.; Yang, Y.; Zhang, S.; Cui, H. Low selenium increases the auxin concentration and enhances tolerance to low phosphorous stress in tobacco. Environ. Exp. Bot. 2018, 153, 127-134. [CrossRef]

66. Haghighi, M.; Sheibanirad, A.; Pessarakli, M. Effects of selenium as a beneficial element on growth and photosynthetic attributes of greenhouse cucumber. J. Plant Nutr. 2016, 39, 1493-1498. [CrossRef]

67. Rezende, R.A.; Rodrigues, F.A.; Soares, J.D.; Silveira, H.R.; Pasqual, M.; Dias, G.D. Salt stress and exogenous silicon influence physiological and anatomical features of in vitro-grown cape gooseberry. Cienc. Rural 2018, 48, e20170176. [CrossRef]

68. Zahedi, S.M.; Abdelrahman, M.; Hosseini, M.S.; Hoveizeh, N.F.; Tran, L.-S.P. Alleviation of the effect of salinity on growth and yield of strawberry by foliar spray of selenium-nanoparticles. Environ. Pollut. 2019, 253, 246-258. [CrossRef]

69. Djanaguiraman, M.; Prasad, P.V.V.; Seppanen, M. Selenium protects sorghum leaves from oxidative damage under high temperature stress by enhancing antioxidant defense system. Plant Physiol. Biochem. 2010, 48, 999-1007. [CrossRef] [PubMed]

70. Nawaz, F.; Ashraf, M.Y.; Ahmad, R.; Waraich, E.A.; Shabbir, R.N. Selenium (Se) regulates seedling growth in wheat under drought stress. Adv. Chem. 2014, 2014, 143567. [CrossRef]

71. Alyemeni, M.N.; Ahanger, M.A.; Wijaya, L.; Alam, P.; Bhardwaj, R.; Ahmad, P. Selenium mitigates cadmium-induced oxidative stress in tomato (Solanum lycopersicum L.) plants by modulating chlorophyll fluorescence, osmolyte accumulation, and antioxidant system. Protoplasma 2018, 255, 459-469. [CrossRef]

72. Nawaz, F.; Naeem, M.; Ashraf, M.Y.; Tahir, M.N.; Zulfiqar, B.; Salahuddin, M.; Shabbir, R.N.; Aslam, M. Selenium supplementation affects physiological and biochemical processes to improve fodder yield and quality of maize (Zea mays L.) under water deficit conditions. Front. Plant Sci. 2016, 7, 1438. [CrossRef]

73. Saffaryazdi, A.; Lahouti, M.; Ganjeali, A.; Bayat, H. Impact of selenium supplementation on growth and selenium accumulation on spinach (Spinacia oleracea L.) plants. Not. Sci. Biol. 2012, 4, 95-100. [CrossRef]

74. Hawrylak-Nowak, B. Effect of selenium on selected macronutrients in maize plants. J. Elem. 2008, 13, 513-519.

75. Stepien, P.; Klbus, G. Water relations and photosynthesis in Cucumis sativus L. leaves under salt stress. Biol. Plant. 2006, 50, 610-616. [CrossRef]

76. Trapp, S.; Feificova, D.; Rasmussen, N.F.; Bauer-Gottwein, P. Plant uptake of $\mathrm{NaCl}$ in relation to enzyme kinetics and toxic effects. Environ. Exp. Bot. 2008, 64, 1-7. [CrossRef]

77. Ma, X.; Zhang, J.; Huang, B. Cytokinin-mitigation of salt-induced leaf senescence in perennial ryegrass involving the activation of antioxidant systems and ionic balance. Environ. Exp. Bot. 2016, 125, 1-11. [CrossRef]

78. Ahanger, M.A.; Agarwal, R.M. Salinity stress induced alterations in antioxidant metabolism and nitrogen assimilation in wheat (Triticum aestivum L) as influenced by potassium supplementation. Plant Physiol. Biochem. 2017, 115, 449-460. [CrossRef] [PubMed]

79. Munns, R.; Tester, M. Mechanisms of Salinity Tolerance. Annu. Rev. Plant Biol. 2008, 59, 651-681. [CrossRef] [PubMed]

80. Guo, C.Y.; Wang, X.Z.; Chen, L.; Ma, L.N.; Wang, R.Z. Physiological and biochemical responses to saline-alkaline stress in two halophytic grass species with different photosynthetic pathways. Photosynthetica 2015, 53, 128-135. [CrossRef]

81. Zhu, J.-K. Plant salt tolerance. Trends Plant Sci. 2001, 6, 66-71. [CrossRef]

82. Mansour, E.; Desoky, E.-S.M.; Ali, M.M.; Abdul-Hamid, M.I.; Ullah, H.; Attia, A.; Datta, A. Identifying drought-tolerant genotypes of faba bean and their agro-physiological responses to different water regimes in an arid Mediterranean environment. Agric. Water Manag. 2021, 247, 106754. [CrossRef]

83. Desoky, E.-S.M.; Mansour, E.; Ali, M.; Yasin, M.A.; Abdul-Hamid, M.I.; Rady, M.M.; Ali, E.F. Exogenously used 24-epibrassinolide promotes drought tolerance in maize hybrids by improving plant and water productivity in an arid environment. Plants 2021, 10, 354. [CrossRef]

84. Desoky, E.-S.M.; Mansour, E.; Yasin, M.A.; El Sobky, E.-S.E.; Rady, M.M. Improvement of drought tolerance in five different cultivars of Vicia faba with foliar application of ascorbic acid or silicon. Span. J. Agric. Res. 2020, 18, 16. [CrossRef]

85. Howladar, S.M. A novel moringa oleifera leaf extract can mitigate the stress effects of salinity and cadmium in bean (Phaseolus vulgaris L.) plants. Ecotoxicol. Environ. Saf. 2014, 100, 69-75. [CrossRef] [PubMed] 
86. Farhangi-Abriz, S.; Torabian, S. Antioxidant enzyme and osmotic adjustment changes in bean seedlings as affected by biochar under salt stress. Ecotoxicol. Environ. Saf. 2017, 137, 64-70. [CrossRef]

87. Elrys, A.S.; Abdo, A.I.; Desoky, E.-S.M. Potato tubers contamination with nitrate under the influence of nitrogen fertilizers and spray with molybdenum and salicylic acid. Environ. Sci. Pollut. Res. 2018, 25, 7076-7089. [CrossRef]

88. Chandrashekar, K.; Sandhyarani, S. Salinity induced chemical changes in Crotalaria striata DC plants. Indian J. Plant Physiol. 1996, $1,44-48$.

89. Chun, S.C.; Paramasivan, M.; Chandrasekaran, M. Proline accumulation influenced by osmotic stress in arbuscular mycorrhizal symbiotic plants. Front. Microbiol. 2018, 9, 2525. [CrossRef]

90. Khan, M.I.R.; Nazir, F.; Asgher, M.; Per, T.S.; Khan, N.A. Selenium and sulfur influence ethylene formation and alleviate cadmiuminduced oxidative stress by improving proline and glutathione production in wheat. J. Plant Physiol. 2015, 173, 9-18. [CrossRef] [PubMed]

91. Sairam, R.K.; Rao, K.V.; Srivastava, G.C. Differential response of wheat genotypes to long term salinity stress in relation to oxidative stress, antioxidant activity and osmolyte concentration. Plant Sci. 2002, 163, 1037-1046. [CrossRef]

92. Şen, A. Oxidative stress studies in plant tissue culture. In Biochemistry, Genetics and Molecular Biology "Antioxidant Enzyme", El-Missiry, M.A., Ed.; World's Largest Science, Technology \& Medicine (INTECH): London, UK, 2012; Volume 3, pp. 59-88.

93. Valero, E.; Macià, H.; Ildefonso, M.; Hernández, J.-A.; González-Sánchez, M.-I.; García-Carmona, F. Modeling the ascorbateglutathione cycle in chloroplasts under light/dark conditions. BMC Syst. Biol. 2015, 10, 11. [CrossRef] [PubMed]

94. Abogadallah, G.M. Insights into the significance of antioxidative defense under salt stress. Plant Signal. Behav. 2010, 5, 369-374. [CrossRef]

95. Rady, M.M.; Varma, C.B.; Howladar, S.M. Common bean (Phaseolus vulgaris L.) seedlings overcome $\mathrm{NaCl}$ stress as a result of presoaking in Moringa oleifera leaf extract. Sci. Hortic. 2013, 162, 63-70. [CrossRef]

96. Desoky, E.-S.M.; Merwad, A.-R.M.; Rady, M.M. Natural biostimulants improve saline soil characteristics and salt stressed-sorghum performance. Comm. Soil Sci. Plant Anal. 2018, 49, 967-983. [CrossRef]

97. Foyer, C.H.; Noctor, G. Ascorbate and Glutathione: The Heart of the Redox Hub. Plant Physiol. 2011, 155, 2-18. [CrossRef]

98. Hasanuzzaman, M.; Nahar, K.; Anee, T.I.; Fujita, M. Exogenous silicon attenuates cadmium-induced oxidative stress in brassica napus 1. By modulating AsA-GSH pathway and glyoxalase system. Front. Plant Sci. 2017, 8, 1061. [CrossRef]

99. Zhou, Y.; Wen, Z.; Zhang, J.; Chen, X.; Cui, J.; Xu, W.; Liu, H.-Y. Exogenous glutathione alleviates salt-induced oxidative stress in tomato seedlings by regulating glutathione metabolism, redox status, and the antioxidant system. Sci. Hortic. 2017, 220, 90-101. [CrossRef]

100. Zhang, Y.; Wang, L.; Liu, Y.; Zhang, Q.; Wei, Q.; Zhang, W. Nitric oxide enhances salt tolerance in maize seedlings through increasing activities of proton-pump and $\mathrm{Na}^{+} / \mathrm{H}^{+}$antiport in the tonoplast. Planta 2006, 224, 545-555. [CrossRef] [PubMed]

101. Shabala, S.; Pottosin, I. Regulation of potassium transport in plants under hostile conditions: Implications for abiotic and biotic stress tolerance. Physiol. Plant. 2014, 151, 257-279. [CrossRef] [PubMed]

102. Gupta, M.; Gupta, S. An overview of selenium uptake, metabolism, and toxicity in plants. Front. Plant Sci. 2017, 7, 2074. [CrossRef]

103. Ahanger, M.A.; Alyemeni, M.N.; Wijaya, L.; Alamri, S.A.; Alam, P.; Ashraf, M.; Ahmad, P. Potential of exogenously sourced kinetin in protecting Solanum lycopersicum from $\mathrm{NaCl}$-induced oxidative stress through up-regulation of the antioxidant system, ascorbate-glutathione cycle and glyoxalase system. PLoS ONE 2018, 13, e0202175. [CrossRef] [PubMed]

104. Alqarawi, A.; Hashem, A.; Abd_Allah, E.; Alshahrani, T.; Huqail, A. Effect of salinity on moisture content, pigment system, and lipid composition in Ephedra alataDecne. Acta Biol. Hung. 2014, 65, 61-71. [CrossRef]

105. Heidari, M.; Tafazoli, E. Effect of sodium chloride on lipoxygenase activity, hydrogen peroxide content and lipid peroxidation rate in the seedlings of three Pistacia rootstocks. J. Agric. Nat. Resour. Sci. 2005, 9, 41-50.

106. Serrano, R.; Mulet, J.M.; Rios, G.; Marquez, J.A.; Larrinoa, I.F.; Leube, M.P.; Mendizabal, I.; Pascual-Ahuir, A.; Proft, M.; Ros, R.; et al. A glimpse of the mechanisms of ion homeostasis during salt stress. J. Exp. Bot. 1999, 50, 1023-1036. [CrossRef]

107. Filek, M.; Keskinen, R.; Hartikainen, H.; Szarejko, I.; Janiak, A.; Miszalski, Z.; Golda, A. The protective role of selenium in rape seedlings subjected to cadmium stress. J. Plant Physiol. 2008, 165, 833-844. [CrossRef]

108. Hawrylak-Nowak, B. Comparative effects of selenite and selenate on growth and selenium accumulation in lettuce plants under hydroponic conditions. Plant Growth Regul. 2013, 70, 149-157. [CrossRef] 